\title{
Semiconducting, biodegradable and bioactive fibers for drug delivery
}

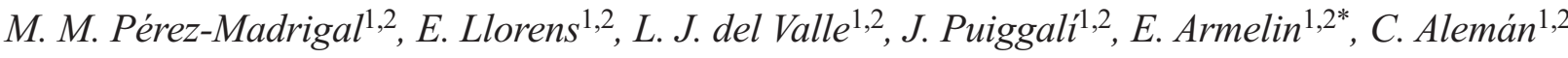 \\ ${ }^{1}$ Departament d'Enginyeria Química, Universitat Politècnica de Catalunya, Av. Diagonal 647, E-08028 Barcelona, Spain \\ ${ }^{2}$ Center for Research in Nano-Engineering, Universitat Politècnica de Catalunya, Campus Sud, Edifici C', C/Pasqual i \\ Vila s/n, E-08028 Barcelona, Spain
}

Received 4 December 2015; accepted in revised form 25 February 2016

\begin{abstract}
In this work we present the drug release properties and morphological studies of fibers formed by mixing different ratios of poly(lactic acid) (PLA) and poly(3-thiophene methyl acetate) (P3TMA) loaded with four drugs (ciprofloxacin, chlorhexidine dihydrochloride, triclosan and ibuprofen sodium salt). Thus, the main aim of this study is to prove that the excellent cellular response of PLA-P3TMA biocompatible scaffolds can be successfully combined with essential applications as drug carrier and delivery systems. Atomic force microscopic (AFM) and scanning electron microscopic (SEM) micrographs of PLA-P3TMA fibers indicate that the presence of the conducting polymer inside the PLA matrix affects the surface morphology, resulting in a significant increment of the bulk conductivity with respect to PLA fibers. Electrospun hybrid fibers of PLA and P3TMA successfully load both hydrophilic and hydrophobic drugs, the release profiles depending on the release environment (i.e. the release rate increases with the hydrophobicity of the medium). Finally, our results prove that the antibacterial activity of the drugs is not affected by their interactions with the PLA-P3TMA matrix.
\end{abstract}

Keywords: biopolymers, biocomposites, conducting polymers, nanotechnology, atomic force microscopy (AFM), drug delivery systems

\section{Introduction}

Research based on the use of bioplastics and biosolvents for the development of advanced materials has increased considerably in the last decade. Poly(lactic acid) (PLA), a 100\% biodegradable biopolymer, has been extensively investigated and used in different commercial and industrial applications, such as automotive parts, clothing and carpet fibers, food packaging, among others. PLA can be synthesized by polycondensation from lactic acid, which is a renewable resource derived from the starch of either corn of sugar beets that is fermented to form glucose and, consequently, converted to lactic acid. Accordingly, PLA can be considered as active player in green chemistry as it reduces significantly the carbon footprint when compared to other oil based traditional plastics. Fur- thermore, due to its good mechanical integrity, biodegradability and biocompatibility, PLA and its copolymers are widely used in biomedical fields. Thus, these materials have been employed for the fabrication of drug loading and release devices, surgical sutures, and scaffolds for tissue and nerve regeneration [1-7].

On the other hand, electroactive conducting polymers (CPs) represent another important family of organic materials with proved biocompatibility $[5,8-$ 14]. More specifically, CPs have attracted the attention of biomedical engineers because cells respond to external electrical and/or electrochemical stimuli in vitro and in vivo when in contact with CPs [12-14]. Thus, application of external potentials has been found to promote cellular adhesion, proliferation,

$\overline{{ }^{*} \text { Corresponding author, e-mail: elaine.armelin@upc.edu }}$

(C) BME-PT 
growth and differentiation. For example, Schmidt and co-workers proved that neurite length of PC-12 cells cultured on polypyrrole (PPy) films increase upon the application of electrical stimuli [15]. These observations suggested that rationally designed conducting and electroactive nanoconstructs (e.g. nanomembranes and nanofibers) could act simultaneously as scaffolds for tissue engineering $[14,15]$ and efficient systems for drug targeting and delivery [16-18]. Indeed, CPs are attractive for a number of biomedical applications as was reviewed by Schmidt and coworkers [19].

One of the major drawbacks in the use of CPs for biomedical applications is the lack of mechanical integrity and restricted processability of many of these materials, combined with their non-degradability [5]. Limitations of CPs are frequently overcome by blending these materials with biodegradable polymers [520]. Albertsson and co-workers have vastly studied the combination of biodegradable polymers with CPs for bioengineering applications [20]. For example, such group studied the degradability and cytotoxicity of blends made of caprolactone and hyperbranched degradable CPs as good candidates for neural tissue engineering application [21]. More recently, freestanding nanomembranes for tissue regeneration were prepared by spin-coating mixtures of polyester [22, 23] or thermoplastic polyurethane [24] with a soluble polythiophene derivative, poly(3-thiophene methyl acetate) (P3TMA). The same approach has also been used to fabricate electroactive biodegradable 3D scaffolds. For example, regular microfibers were recently obtained by electrospinning a mixture of P3TMA and biodegradable poly(ester urea) [25]. Overall the results obtained in this field proved the synergy associated with the combination of biodegradable polymers and CPs, their mixtures showing biodegradability and both electrochemical and electrical activities.

In the last years some studies based on nanofibers made of PLA-CP blends have been reported. However, the preparation of such nanoconstructs has been essentially restricted to the combination of biodegradable polymer with small electroactive oligomers (e.g. oligothiophenes [26] and oligoanilines $[27,28]$, polyaniline (PAni) or PPy derivatives [20, 29-31]. On the other hand, antecedents on drug delivery systems fabricated with nanofibers based on biodegradable polymers-CP blends are very scarce. Martin and co-workers developed electrospun poly (lactide-co-glycolide)-poly(3,4-ethylenedioxythiophene) conductive core-sheath nanofibers (PLGAPEDOT) able to modulate the release of dexamethasone, an anti-inflammatory drug for the central nervous system [32]. More recently, we designed and synthesized series of linear micro/nanofibers for tissue engineering applications using PLA-P3TMA blends with different compositions [33]. Scaffolds containing P3TMA exhibited enhanced cellular proliferation and adhesion in comparison to those made of pristine PLA. Such improvement was attributed to the electrochemical properties (i.e. ability to exchange ions) and wettability properties provided by the CP.

The objective of the present work is to show the multifunctionality of scaffolds made of PLA-P3TMA fibers, which is based on the combination of the already known cellular response [33] with their high activity as drug carrier and delivery systems. In order to prove this duality, the encapsulation and delivery of different drugs in PLA-P3TMA systems have been investigated by controlling both the fiber morphology and the composition of the blend. Four drugs have been considered for this purpose: ciprofloxacin (CIP), chlorhexidine dihydrochloride (CHX), triclosan (TCS), and ibuprofen sodium salt (IBU). The selection of these compounds was based on the fact they are typically considered as model antibiotics (CIP), biocides (CHX and TCS) and anti-inflammatories (IBU).

\section{Experimental section}

\subsection{Materials}

3-Thiophene acetic acid (3TAA) $(98.0 \%)$ was purchased from Fluka (Sigma-Aldrich). Iron chloride anhydrous (97.0\%), dry methanol (99.5\%) and chloroform (99.9\%) were purchased from Panreac Quimica S.A.U. (Spain) and used as received without further purification. PLA, a product of Natureworks (polymer 2002D), was kindly supplied by Nupik International (Polinyà, Spain). According to the manufacturer, this PLA has a D-lactide content of $4.25 \%$, a residual monomer content of $0.3 \%$, a density of $1.24 \mathrm{~g} / \mathrm{cc}$, a glass transition temperature $\left(T_{\mathrm{g}}\right)$ of $58^{\circ} \mathrm{C}$, a melting point of $153^{\circ} \mathrm{C}$ and molecular weights of $M_{\mathrm{n}}=59300 \mathrm{~g} \cdot \mathrm{mol}^{-1}$ and $M_{\mathrm{W}}=117500 \mathrm{~g} \cdot \mathrm{mol}^{-1}$. CIP ( $\geq 98 \%$ HPLC), CHX ( $\geq 98 \%$ ), TCS (irgasan, $\geq 97 \%$ HPLC), and IBU ( $\geq 98 \%$ GC) were purchased from Sigma-Aldrich. Escherichia coli CECT 101 and Staphylococcus epidermidis CECT 231 bacterial strains 
were obtained from the Spanish Collection of Type Culture (Valencia, Spain), and culture media were purchased from Difco (Detroit, MI, USA).

\subsection{Synthesis of poly(3-thiophene methyl acetate)}

P3TMA was synthesized by chemical oxidative coupling polymerization in dry chloroform following the procedure described by Kim et al. [34], which was successfully used in previous works [22-25]. Anhydrous ferric chloride $\left(\mathrm{FeCl}_{3}\right)$ was used as both oxidant and dopant. The monomer 3-thiophene methyl acetate (3TMA) was obtained with a $74 \%$ yield by refluxing 3TAA in dry methanol for 24 hours at a temperature of $90^{\circ} \mathrm{C}$. P3TMA was subsequently prepared by a chemical oxidative coupling polymerization in dry chloroform and using anhydrous ferric chloride $\left(\mathrm{FeCl}_{3}\right)$ as oxidant and dopant. The polymerization yield was $c a .61 \%$ after removing the residual oxidant oligomers. Molecular weights were estimated by size exclusion chromatography (SEC) using 1,1,1,3,3,3-hexafluoroisopropanol as eluent. The number and weight average molecular weights found were $M_{\mathrm{n}}=10700 \mathrm{~g} \cdot \mathrm{mol}^{-1}$ and $M_{\mathrm{w}}=22500 \mathrm{~g} \cdot \mathrm{mol}^{-1}$ for P3TMA. ${ }^{1} \mathrm{H}-\mathrm{NMR}\left(400 \mathrm{MHz}, \mathrm{CDCl}_{3}\right) \delta=7.28$ 7.07 (m, 1H, Ar-H), 3.68(s, 2H, $\left.-\mathrm{CH}_{2}-\right), 3.63$ (s, 3H, $\left.\mathrm{O}-\mathrm{CH}_{3}\right) ;{ }^{13} \mathrm{C}-\mathrm{NMR}\left(100 \mathrm{MHz}, \mathrm{CDCl}_{3}\right) \delta=170.8$ $(\mathrm{C}=\mathrm{O}), 136-124(\mathrm{Ar}-\mathrm{C}), 52.1\left(\mathrm{O}-\mathrm{CH}_{3}\right), 34.3\left(\mathrm{CH}_{2}\right)$; FTIR-ATR $\left(\mathrm{cm}^{-1}\right)$ : 3095-3010 $(=\mathrm{C}-\mathrm{H} \beta$, thiophene ring), $2998(\mathrm{C}-\mathrm{H}$ aliphatic $), 1735(\mathrm{C}=\mathrm{O}$, ester $), 1436$ $\left(-\mathrm{CH}_{2}-\right), 1375$ (-O-CH 3 , ester $), \quad 1255-1160$ (C-O- $\mathrm{CH}_{3}$, ester), $840(\mathrm{C}-\mathrm{H} \beta$, thiophene ring).

\subsection{Preparation of PLA-P3TMA fibers}

For the nanofiber preparation, mixtures of PLA and P3TMA were electrospun from chloroform/acetone $(2: 1 \mathrm{v} / \mathrm{v})$ at polymer concentrations of $5 \mathrm{w} / \mathrm{v} \%$ and $0-5 \mathrm{w} / \mathrm{v} \%$ for PLA and P3TMA, respectively. Samples will be identified indicating the PLA and P3TMA ratio. According to our previous optimization of the processing conditions [33], PLA electrospun fibers were collected on a target with a deposition distance of $12 \mathrm{~cm}$, applying a voltage of $20 \mathrm{kV}$ with a portable High Voltage Source (model ES30-5W, Gamma High Voltage Research, Ormond Beach (FL), USA) and a flow rate of $4 \mathrm{~mL} \cdot \mathrm{h}^{-1}$. The voltage and flow-rate used to prepare PLA-P3TMA fibers was $25 \mathrm{kV}$ and $4 \mathrm{~mL} \cdot \mathrm{h}^{-1}$, respectively. Polymer solutions were delivered via a KDS100 infusion syringe pump (code KDS100, KD Scientific Inc., Holliston (MA), USA) to control the mass-flow rate, and the tip used had an inside diameter of $0.84 \mathrm{~mm}$ (syringe needle 18G). All electrospinning experiments were carried out at room temperature. The diameter of the fibers prepared under such conditions ranged from 600 to $800 \mathrm{~nm}$.

\subsection{PLA-P3TMA (2:1 ratio) fibers drug-loading}

PLA $(0.5 \mathrm{~g})$ was dissolved in $5 \mathrm{~mL}$ of chloroform-acetone mixture $(2: 1 \mathrm{v} / \mathrm{v})$ and P3TMA $(0.25 \mathrm{~g})$ in $4 \mathrm{~mL}$ of the same solvent mixture. Then, $1 \mathrm{~mL}$ of dimethylsulfoxide (DMSO) containing the drug $(0.1 \mathrm{~g})$ was added, and the mixture was homogenized by vortexing to obtain an electrospinnable solution of $5 \mathrm{w} / \mathrm{v} \%$ PLA, $2.5 \mathrm{w} / \mathrm{v} \%$ P3TMA and $1 \mathrm{w} / \mathrm{v} \%$ drug. The electrospinning process was conducted at room temperature, fibers being collected at a distance of $12 \mathrm{~cm}$ from the needle ( $18 \mathrm{G}$, inside diameter of $0.84 \mathrm{~mm}$ ). The applied voltage and the flow rate was of $25 \mathrm{kV}$ and $4 \mathrm{~mL} \cdot \mathrm{h}^{-1}$, respectively.

\subsection{Characterization}

${ }^{1} \mathrm{H}-\mathrm{NMR}$ and ${ }^{13} \mathrm{C}$-NMR spectra were acquired with a Bruker AMX-300 spectrometer (Bruker Corporation, Billerica (MA), USA) operating at $300.1 \mathrm{MHz}$. Chemical shifts were calibrated using tetramethylsilane as an internal standard. Deuterated chloroform was used as the solvent.

Infrared absorption spectra were recorded with a Fourier Transform FTIR 4100 Jasco spectrometer (Jasco Analytical Instruments, Easton, (MD), USA) in the $4000-600 \mathrm{~cm}^{-1}$ range. An attenuated total reflection (ATR) system with a heated Diamond ATR TopPlate (model MKII Golden Gate ${ }^{\mathrm{TM}}$, Specac Ltd., Orpington (Kent), UK) was used.

Atomic force microscopy (AFM) images were obtained for all the samples in tapping mode with an AFM Dimension 3100 microscope and a NanoScope ${ }^{\circledR}$ $\mathrm{V}$ controller (Veeco Instruments Inc., Plainview (NY), USA) at ambient conditions. A silicon probe (model Tap150-G, Budget Sensors ${ }^{\circledR}$, Innovative Solutions Bulgaria Ltd., Sofia, Bulgaria) with a resonant frequency and spring constant of $150 \mathrm{kHz}$ and $5 \mathrm{~N} \cdot \mathrm{m}^{-1}$, respectively, was used. The row scanning frequency was set at $0.8 \mathrm{~Hz}$ and the scan window size varied from $1 \times 1 \mu \mathrm{m}^{2}$ to $20 \times 20 \mu \mathrm{m}^{2}$. Data were acquired using the Reasearch NanoScope software (v. 7.30) and, afterwards, they were analyzed using the NanoScope Analysis analysis software (v. 1.20). 
The Root Mean Square (RMS) roughness, which is the average height deviation taken from the mean data plane, was determined using the statistical application of the NanoScope Analysis software (1.20, Veeco).

A Confocal Raman-AFM microscope (model a300R+, WITec - Wissenschaftliche Instrumente und Technologie GmbH-, Ulm, Germany) was used to collect Raman spectra from the homopolymers, PLAP3TMA blends and drug loaded samples. For excitation, a laser with wavelength of $785 \mathrm{~nm}$ was used. The integration time was between 0.3 and $2.1 \mathrm{sec}-$ onds depending on the scan, and high resolution Raman images were obtained by collecting complete Raman spectra at less than $3 \mathrm{~cm}^{-1}$ per pixel. Fibers were analyzed as obtained after the electrospining process, without any additional preparation, at room temperature and after the exposure time necessary to decay the fluorescence.

The number-average molecular weight $\left(M_{\mathrm{n}}\right)$ and weight-average molecular weight $\left(M_{\mathrm{w}}\right)$ were measured by gel permeation chromatography (GPC) at $25^{\circ} \mathrm{C}$ using a chromatograph (model 1525 - Binary HPLC Pump) equipped with a refractive index detector (model 2414) from Waters Cromatografía, S.A., (Cerdanyola del Vallès, Spain). GPC measurements were carried out using tetrahydrofuran (THF) as eluent with a flow rate of $1.0 \mathrm{~mL} \cdot \mathrm{min}^{-1}$, respectively. Polystyrene standards were used for calibration.

Optical morphologic observations were performed using Zeiss Axioskop 40 microscope (Carl Zeiss Microscopy, LLC, USA). Micrographs were taken with Zeiss AxiosCam MRC5 digital camera (Carl Zeiss Microscopy, LLC, USA). Morphological characterization of the electrospun samples was conducted by scanning electron microscopy (SEM) using a Focus Ion Beam Zeiss Neon 40 instrument (Carl Zeiss, Germany). Prior sample observation, carbon coating was accomplished by using a Sputter Coater (model Mitec K950, Quorum Technologies Ltd., Ashford (Kent), UK) fitted with a film thickness monitor $k 150 \times$. Samples were visualized at an accelerating voltage of $5 \mathrm{kV}$. Diameter of electrospun fibers was measured with the SmartTiff software from Carl Zeiss SMT Ltd (Jena, Germany).

Contact angle (CA) measurements were performed using an OCA 15EC (DataPhysics Instruments $\mathrm{GmbH}$, Filderstadt, Germany) equipment and using the sessile droplet method at room temperature. Three dif- ferent polar solvents were tested: ultrapure water, formamide and ethylene glycol. For the static contact angle (sCA) measurements, $0.5 \mu \mathrm{L}$ droplets of liquid were dispensed on the respective surfaces. Images were recorded after drop stabilization (30 s) using the SCA 20 software. CA values were obtained as the average of fourteen independent measures for each sample. The surface energy (SE) of PLAP3TMA samples was calculated based on the SCA data by applying several mathematical approaches: Equation-of-State (EoS), Fowkes, Owens-WendtRabel-Kaelble (OWRK) and Wu models. Finally, the work of adhesion $\left(W_{\mathrm{A}}\right)$ was determined to quantify the wettability of a liquid droplet on the sample.

\subsection{Electrochemical impedance spectroscopy} (EIS)

EIS measurements were performed using a potentiostat/galvanostat (model AUTOLAB PGSTAT302N, Metrohm AG, Herisau, Switzerland) in the $100 \mathrm{kHz}$ to the $10 \mathrm{mHz}$ frequency range and the amplitude of the sinusoidal voltage was $10 \mathrm{mV}$. All experiments were carried at room temperature. The fiber mats were cut in a disc format $\left(1.766 \mathrm{~cm}^{2}\right)$ and were sandwiched between two stainless steel electrodes assembled into an isolating resin holder [35]. The cell was tightened with screws to ensure constant pressure fastening. The thickness of the films, which was determined by a micrometer, varied between 18.9 and $26.0 \mu \mathrm{m}$. Prior to cell closing, samples were immersed in phosphate buffer saline (PBS, $\mathrm{pH}$ 7.4) at room temperature for $24 \mathrm{~h}$ and the water excess wiped out with a tissue. After data collection, EIS results were then processed and fitted to an electrical equivalent circuit (EEC).

\subsection{Drug-release experiments}

Drug-loaded mats were cut into small square pieces $\left(20 \times 20 \times 0.1 \mathrm{~mm}^{3}\right)$ which were weighed and placed into polypropylene tubes. PBS and PBS supplemented with $70 \mathrm{v} / \mathrm{v} \%$ of ethanol (PBS-EtOH) were considered as release media. The addition of ethanol to hydrophilic PBS increases the hydrophobicity of the medium and provokes some swelling effect, both favoring the release of hydrophobic drugs as TCS [36]. The release of TCS from PLA fibers in PBS containing 10 and $70 \mathrm{v} / \mathrm{v} \%$ of ethanol was found to be 40 and $98 \%$ after $48 \mathrm{~h}$, respectively, indicating that the composition of PBS-EtOH is adequate to alter the equilibrium defined by the affinity of PLA towards 
hydrophobic drugs [36]. Drug-release assays were carried out by immersing sample mats in $50 \mathrm{~mL}$ of the release medium at $25^{\circ} \mathrm{C}$ for 1 week. Aliquots $(1 \mathrm{~mL})$ were drawn from the release medium at predetermined intervals, and an equal volume of fresh medium was added to the release vessel. The drug concentration in the release medium was evaluated by UV-vis spectroscopy (UV-VIS-NIR Spectrophotometer model UV-3600 Plus from Shimadzu Corporation, Kyoto, Japan). Calibration curves were obtained by plotting the absorbance measured at the corresponding wavelengths (i.e. 322, 254, 281, and $260 \mathrm{~nm}$ for CIP, CHX, TCS and IBU, respectively) against drug concentration. Finally, the mats were dissolved in chloroform and the residual drug was extracted in ethanol for quantification. All tests were performed in triplicate to control the homogeneity of the release, and the results were averaged.

\subsection{Agar diffusion test}

Escherichia coli and Staphylococcus epidermidis were cultured aerobically overnight in $15 \mathrm{~mL}$ of brain-heart infusion (BHI) broth at $37^{\circ} \mathrm{C}$. The bacterial suspension was spread on Plate Count Agar (PCA) (Difco ${ }^{\mathrm{TM}}$ MI Agar, from Becton, Dickinson and Company, Franklin Lakes (NJ), USA) using a nylon swab. Uniform disks of $1 \mathrm{~mm}$ thick and $5 \mathrm{~mm}$ in diameter were prepared by perforating the drugloaded PLA-P3TMA electrospun mats. Then, samples were placed on the surface of the agar plates and incubated at $37^{\circ} \mathrm{C}$. After $24 \mathrm{~h}$, the inhibition zone around each specimen was observed to determine the activity of the drug.

\section{Results and discussion}

\subsection{Topography and RAMAN analyses of PLA-P3TMA fibers}

The electrospinning conditions and morphology of PLA-P3TMA fiber mats were examined in our previous study [33]. Scanning electron microscopy (SEM) micrographs evidenced that the incorporation of P3TMA in the electrospinning solution induced some structural changes in the PLA fiber: (1) the fiber diameter decreased slightly; (2) the fiber surface became smoother; and (3) heterogeneous clusters made of P3TMA aggregates appeared inside the fibers. Furthermore, both the electrochemical activity and the bioactivity of the fibers increased with the content of P3TMA.
AFM analyses (Figure 1) provided more precise information about the roughness of electrospun PLA and of PLA-P3TMA fibers. PLA fibers exhibit remarkable irregularities on their surface, their average RMS roughness \pm 2 times the corresponding standard deviation (calculated using five different samples) being $41.4 \pm 3.8 \mathrm{~nm}$. The average RMS roughness determined for 5:1, 2:1 and 1:1 PLA-P3TMA compositions (Figures $1 \mathrm{~b}-\mathrm{d}$ ) is $34.8 \pm 2.5,39.9 \pm 3.0$ and $35.6 \pm 4.5 \mathrm{~nm}$, respectively. AFM phase images allow us to distinguish a phase contrast for PLA-P3TMA samples that is not observed for the PLA fibers. This supports that the two polymers mixed in the chloroform solution successfully incorporate into the electrospun fibers, even though it is not possible to associate each region (i.e. dark or bright areas) to a specific polymer-rich phase.

Further studies were carried out to understand P3TMA compositional distribution on PLA-P3TMA fibers by means of Raman spectroscopy. Figure 2a shows optical images taken with the Raman confocal microscope of PLA fibers, the Raman spectrum being displayed in Figure 2b. As it can be seen, the PLA spectrum presents identifiable peaks at well-known positions [37]. The most intense band, which corresponds to the $v \mathrm{C}-\mathrm{COO}$ stretching is located at $c a$. $874 \mathrm{~cm}^{-1}$ (Figure 2b).

Optical images of PLA-P3TMA fibers are displayed in Figures 2c and 2e. P3TMA powder was analyzed by Raman spectroscopy and the resulting spectrum is depicted in Figure 2d). As the fluorescence induced by the laser at $532 \mathrm{~nm}$ used for PLA fibers is very high for P3TMA, CP-containing samples were analyzed using the laser at $785 \mathrm{~nm}$. This leaded to less fluorescence overlapping but also to a lower peak resolution. The most intense line for the P3TMA spectra is identified at $c a .1480 \mathrm{~cm}^{-1}$, which corresponds to the totally symmetric in-phase vibration of the thiophene rings spread over the polymer chains [38]. Raman spectra were recorded from three different spots of the PLA-P3TMA fibers (Figures $2 \mathrm{c}$ and 2d) as well as from P3TMA particles, which are clearly visible as dark spots inside the fibers (Figures $2 c$ and $2 \mathrm{e}$ ). These results corroborate the homogenous distribution of P3TMA among the PLA fibers, even though some CP agglomerates are also detected. Moreover, 2:1 PLAP3TMA fibers loaded with drugs were also evaluated by Raman spectroscopy (discussed below). 

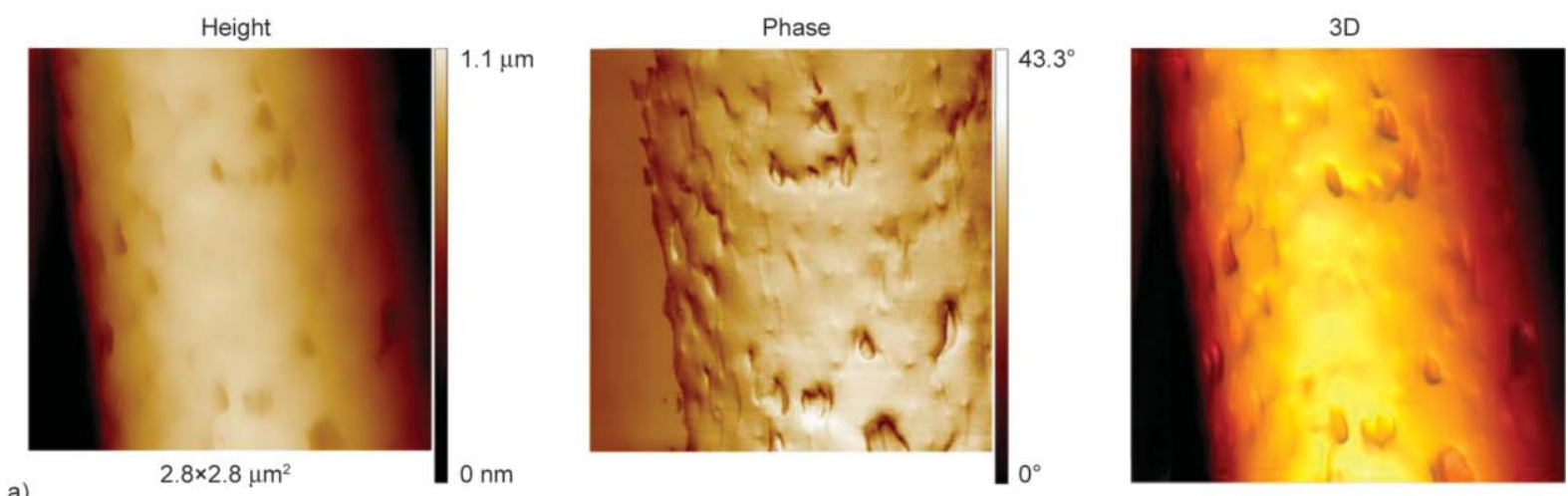

a)
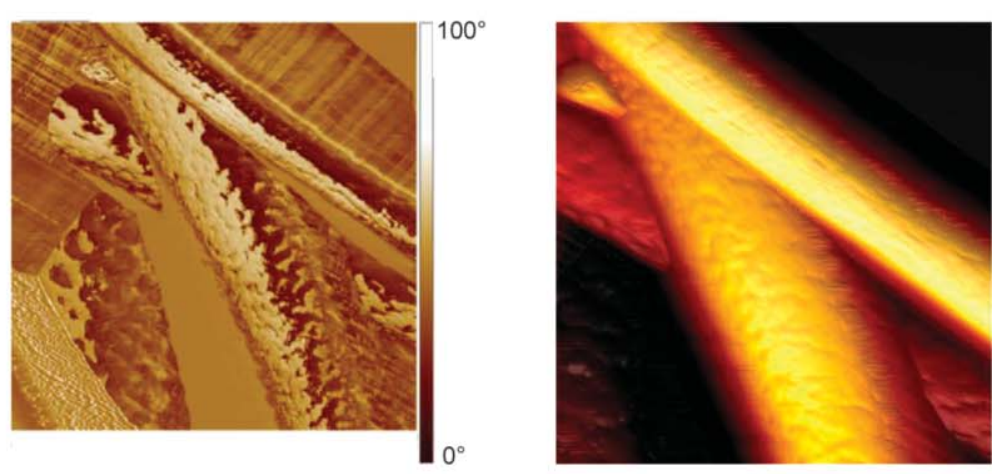

b)
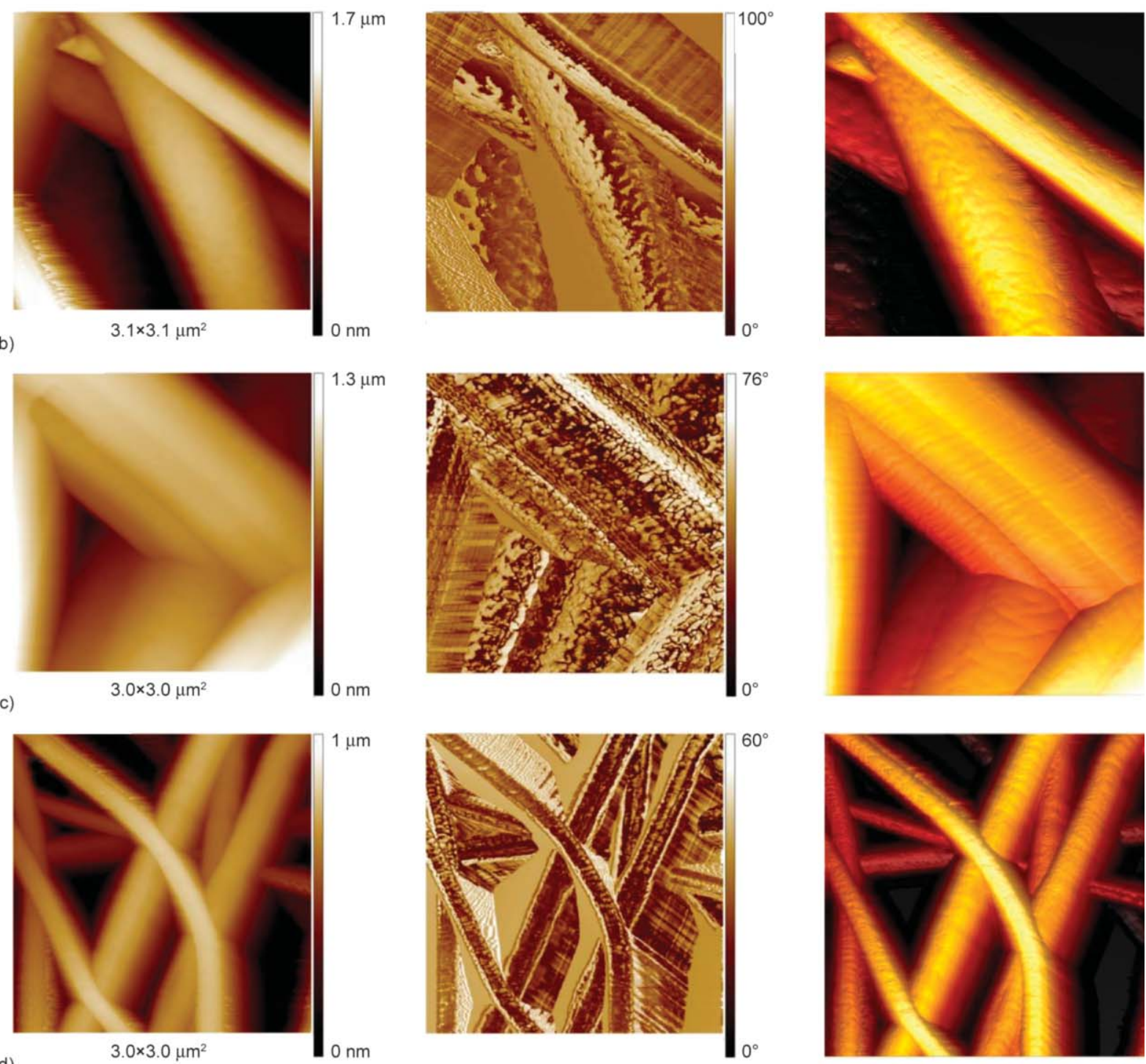

d)

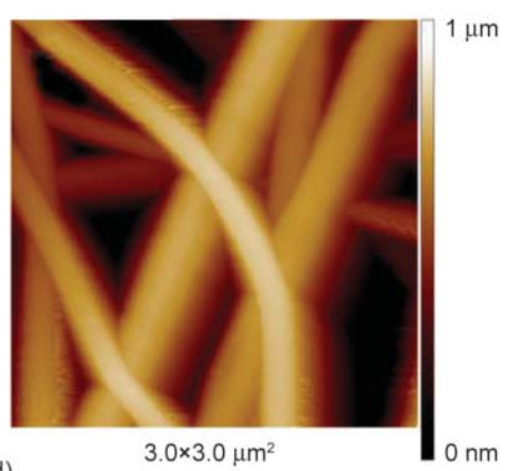

Figure 1. AFM micrographs from PLA and PLA-P3TMA fibers scaffolds prepared by electrospinning: (a) PLA, (b) 5:1 PLA-P3TMA, (c) 2:1 PLA-P3TMA and (d) 1:1 PLA-P3TMA

\subsection{Wettability and surface energy determination}

Understanding of the wetting properties of PLAP3TMA fibers is crucial for their use as drug carrier and delivery systems. Two wetting states have been defined for hydrophobic surfaces [39]. In the Wenzel state, which corresponds to a homogeneous wetting regime, the liquid completely penetrates into the grooves defined by the surface roughness, while air bubbles are inside the grooves underneath the liquid in the heterogeneous wetting regime associated with the Cassie-Baxter state. Air entrapped into 3D fi- 


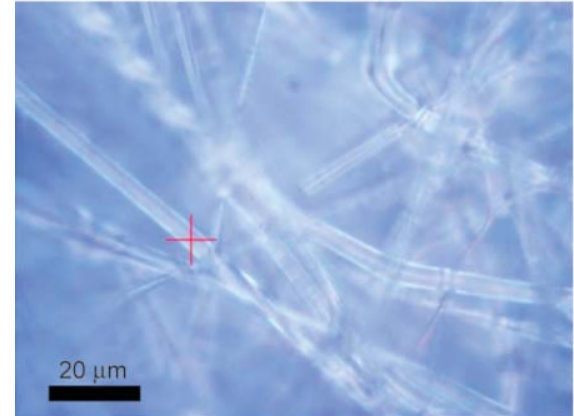

a)

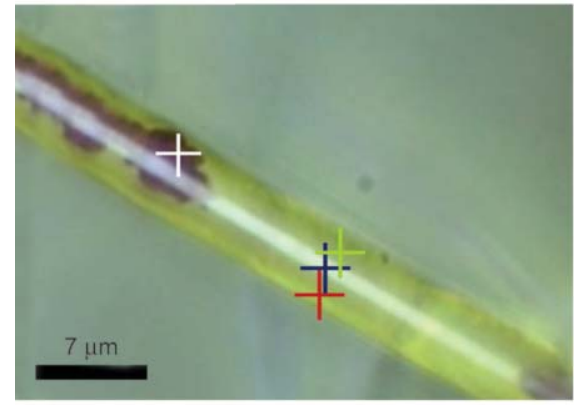

c)

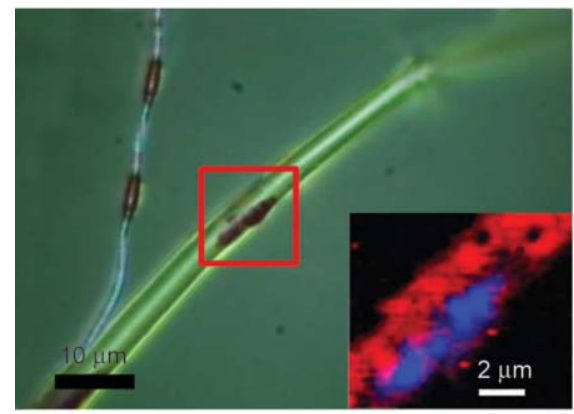

e)
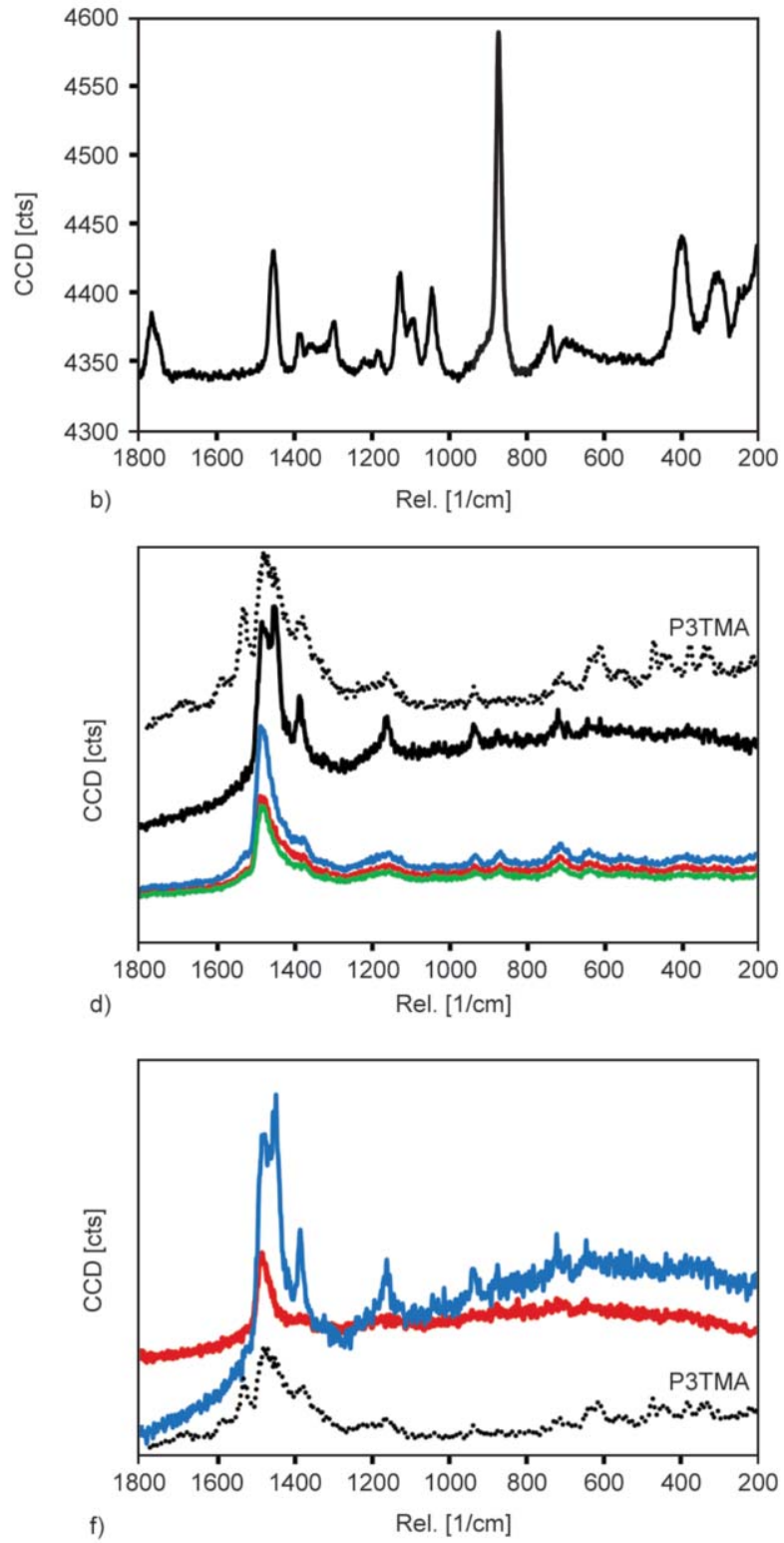

Figure 2. (a) PLA fiber optical image, (b) PLA Raman spectra at the cross point from (a). (c) PLA-P3TMA (2:1) fiber optical image. (d) Raman spectra at the cross points from (b). The solid black line corresponds to the white cross in (c) and the discontinued line to powder P3TMA. (e) PLA-P3TMA (2:1) fiber optical image. Inset: scanned area (red box). (f) Raman spectra associated with the inset in (e).

brous scaffolds may act as a barrier precluding water penetration and, therefore, affecting the drug-delivery. However, a transition from the Cassie-Baxter state to the Wenzel state may occur whenever air regions are no longer thermodynamically stable and liquid infiltrates the surface. Thus, the drug-delivery rate can be tuned by controlling the wettability of drug-loaded systems [40].

Table 1 gives the static contact angles (sCA) values of deionized water, formamide and ethylene glycol solvents determined for PLA and PLA-P3TMA scaffolds. Results are compared with those obtained for films prepared by solvent-casting a chloroform solution of the corresponding blend. As it can be seen, the wetting behavior depends on the organization. For each solvent, the sCA of scaffolds made of fiber diameters varying from 600 to $800 \mathrm{~nm}$ is higher than that of films prepared by solvent evaporation for PLA and all blend compositions. Hence, the fiber matrix is able to block the liquid phase resulting in a less wettable surface (Figure 3), which has been attributed to both the fiber roughness and the air entrapped at the surface. In spite of this, the exact final frontier of the three-phase contact line (TPCL) is unclear since the liquid may penetrate the porous matrix through the space left among adjacent fibers. Therefore, it is not possible to ascertain which state (i.e. Wenzel, Cassie-Baxter or the transition between them) is the 
Table 1. Static contact angle values (in degrees) for PLA and PLA-P3TMA scaffolds prepared by electrospinning and for films obtained by solvent casting, respectively

\begin{tabular}{|l|c|c|c|c|c|c|}
\hline \multirow{2}{*}{ Composition } & \multicolumn{2}{|c|}{ Water $^{\mathbf{a}}$} & \multicolumn{2}{c|}{ Formamide $^{2}$} & \multicolumn{2}{c|}{ Ethylene glycol } \\
\cline { 2 - 7 } & Scaffolds & Solvent casting $^{\mathbf{b}}$ & Scaffolds & Solvent casting $^{\mathbf{b}}$ & Scaffolds $^{\text {Solvent casting }}{ }^{\mathbf{b}}$ \\
\hline PLA & $132.0 \pm 2.2$ & $76.1 \pm 3.1$ & $121.8 \pm 5.4$ & $55.3 \pm 2.2$ & $110.5 \pm 2.7$ & $50.0 \pm 2.4$ \\
\hline PLA-P3TMA 5:1 & $130.5 \pm 2.1$ & $90.5 \pm 3.4$ & $118.7 \pm 3.2$ & $56.0 \pm 2.3$ & $123.8 \pm 3.8$ & $55.8 \pm 2.1$ \\
\hline PLA-P3TMA 2:1 & $135.3 \pm 1.5$ & $86.9 \pm 5.2$ & $121.6 \pm 4.7$ & $55.4 \pm 3.2$ & $130.1 \pm 2.0$ & $55.0 \pm 3.4$ \\
\hline PLA-P3TMA 1:1 & $134.6 \pm 2.4$ & $87.7 \pm 3.8$ & $127.4 \pm 2.4$ & $68.6 \pm 5.6$ & $126.7 \pm 2.0$ & $54.9 \pm 1.6$ \\
\hline
\end{tabular}

${ }^{a}$ Taken from reference 33. ${ }^{\text {bLA }}$ and PLA-P3TMA films were solvent casted from a solution of $16 \mathrm{mg} \cdot \mathrm{mL}^{-1}$ of polymer in $\mathrm{CHCl}_{3}$ solvent. Mean values correspond to the average of at least 14 droplets of water, formamide and ethylene glycol.

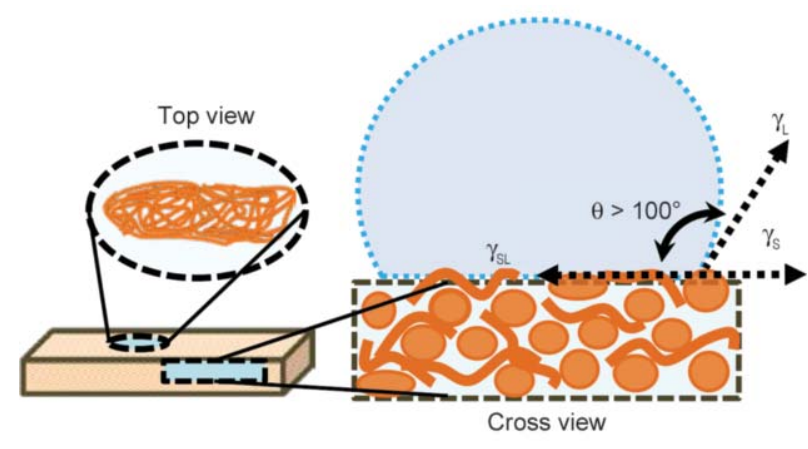

Figure 3. Expected wetting behavior of PLA-P3TMA scaffold samples where air pockets avoid the liquid infiltration

most appropriated to adjust the wetting behavior of PLA and PLA-P3TMA fibrous scaffolds.

Also, sCA values of water and formamide indicate that the incorporation of P3TMA into PLA fibers does not provoke significant changes in the wettability of the polyester (Table 1), independently of the blend composition. For ethylene glycol, PLA-P3TMA fibers exhibit lower wettability than PLA, the sCA of the polyester increasing $13-20^{\circ}$ upon the addition of the CP. This is a very attractive result for drug-release applications since the low wettability of PLAP3TMA scaffolds in such solvents is expected to be accompanied by a slow release.

Surface phenomena, like wetting, adsorption and adhesion, are controlled by the surface energy (SE) of the materials used for deposition. The sCA values displayed in Table 1 have been used to estimate the SE using the Equation-of-State (EoS) [41], Fowkes [42], and Owens-Wendt-Rabel-Kaelble (OWRK) [43, 44] theoretical models. As each model considers differ- ent physical assumptions and interactions for the calculation of the SE components, comparison among such approaches is not an easy task. Results derived from the different models, which are displayed in Table 2, reveal a general tendency. The SE of the solidgas interface $\left(\gamma_{\mathrm{S}}\right)$ and its dispersion and polar components $\left(\gamma_{\mathrm{s}}{ }^{\mathrm{d}}\right.$ and $\gamma_{\mathrm{s}}^{\mathrm{p}}$, respectively) decrease with increasing P3TMA content. Samples with high SE, like PLA fibers, interact more readily with liquids because of their bonding potential. Results allow us to conclude that a small percentage of P3TMA adequately dispersed in the electrospinning process is able to reduce the $\gamma_{\mathrm{S}}$ components, precluding rapid drug release.

The influence of the fibrous morphology in the SE of PLA and PLA-P3TMA was examined by comparing results displayed in Table 2 with those obtained for films prepared by solvent casting using chloroform solutions (Table 3). As it can be seen, the $\gamma_{\mathrm{S}}$ was one order of magnitude higher for films than for fibers. For PLA films, the $\gamma_{\mathrm{S}}$ values displayed in Table 3 are in good agreement with those reported in the literature, which range from 35.1 to $50 \mathrm{~mJ} \cdot \mathrm{m}^{-2}$ (up to $50 \mathrm{~mJ} \cdot \mathrm{m}^{-2}$ ), with $\gamma_{\mathrm{s}}{ }^{\mathrm{d}} c a$. 26.9-29.7, and $\gamma_{\mathrm{s}}{ }^{\mathrm{p}} c a$. $8.2-10.5 \mathrm{~mJ} \cdot \mathrm{m}^{-2}$ [45]. Similarly, the SE values of PLA-P3TMA interfaces and, therefore, their wetting response, are influenced by their morphology.

The highest work of adhesion $\left(W_{\mathrm{A}}\right)$, calculated using the results derived from the OWRK model, was obtained for PLA scaffolds, which was attributed to the fact that PLA exhibits the highest $\gamma_{\mathrm{S}}$ (Table 2). Accordingly, this surface is less repellent to liquid than

Table 2. Surface energy parameters and $W_{\mathrm{A}}$ values $\left[\mathrm{mJ} \cdot \mathrm{m}^{-2}\right]$ for PLA-P3TMA scaffold samples

\begin{tabular}{|l|c|c|c|c|c|c|c|c|}
\hline \multirow{2}{*}{} & EoS & Fowkes & \multicolumn{3}{|c|}{ OWRK } & \multicolumn{3}{c|}{ Work of adhesion $\left.{ }^{\mathbf{a}} \boldsymbol{W}_{\mathbf{A}}\right)$} \\
\cline { 2 - 9 } & $\gamma_{\mathbf{S}}$ & $\gamma_{\mathbf{s}}{ }^{\mathbf{1}}$ & $\gamma_{\mathbf{s}}{ }^{\mathbf{d}}$ & $\gamma_{\mathbf{s}}^{\mathbf{p}}$ & $\gamma_{\mathbf{S}}$ & Water & Formamide & Ethylene glycol \\
\hline PLA & $7.07 \pm 0.26$ & $7.09 \pm 0.23$ & $10.99 \pm 0.00$ & $0.62 \pm 0.00$ & $11.61 \pm 0.00$ & 49.08 & 49.02 & 44.72 \\
\hline PLA-P3TMA 5:1 & $6.53 \pm 0.36$ & $5.05 \pm 0.21$ & $3.95 \pm 0.00$ & $0.11 \pm 0.00$ & $4.06 \pm 0.00$ & 34.68 & 33.18 & 30.23 \\
\hline PLA-P3TMA 2:1 & $5.02 \pm 0.38$ & $3.51 \pm 0.28$ & $3.61 \pm 0.00$ & $0.00 \pm 0.00$ & $3.62 \pm 0.00$ & 33.72 & 33.13 & 29.26 \\
\hline PLA-P3TMA 1:1 & $4.43 \pm 0.18$ & $3.61 \pm 0.14$ & $2.52 \pm 0.00$ & $0.13 \pm 0.00$ & $2.65 \pm 0.00$ & 30.37 & 28.45 & 25.91 \\
\hline
\end{tabular}

${ }^{\mathrm{a}} W_{\mathrm{A}}$ values for PLA-P3TMA scaffolds based on data derived from the OWRK model. 
Table 3. Surface energy parameters of PLA and PLA-P3TMA samples prepared by solvent casting

\begin{tabular}{|c|c|c|c|c|c|}
\hline & EoS & Fowkes & \multicolumn{3}{|c|}{ OWRK } \\
\hline PLA-P3TMA & $\gamma_{\mathbf{S}}$ & $\gamma_{\mathbf{s}} \mathbf{d}^{\mathbf{d}}$ & $\boldsymbol{\gamma}_{\mathbf{s}}^{\mathbf{d}}$ & $\gamma_{\mathbf{s}}^{\mathbf{p}}$ & $\gamma_{\mathbf{S}}$ \\
\hline $1: 0$ & $37.65 \pm 0.24$ & $35.99 \pm 0.02$ & $33.99 \pm 0.03$ & $4.42 \pm 0.01$ & $38.41 \pm 0.03$ \\
\hline $5: 1$ & $33.79 \pm 0.77$ & $53.67 \pm 0.10$ & $73.08 \pm 0.00$ & $2.31 \pm 0.01$ & $75.39 \pm 0.00$ \\
\hline $2: 1$ & $34.75 \pm 0.69$ & $54.60 \pm 0.10$ & $58.20 \pm 0.02$ & $0.10 \pm 0.00$ & $58.31 \pm 0.02$ \\
\hline $1: 1$ & $32.04 \pm 0.38$ & $47.93 \pm 0.10$ & $37.66 \pm 0.02$ & $0.95 \pm 0.00$ & $39.62 \pm 0.02$ \\
\hline
\end{tabular}

systems with $\mathrm{CP}$. In conclusion, the $\mathrm{SE}$ and $W_{\mathrm{A}}$ of PLA-P3TMA fibrous scaffolds prepared by electrospinning are substantially lower than those obtained for film. Thus, the surface of the fibers is more repellent to polar liquids than that of films.

\subsection{Electrochemical impedance spectroscopy} The presence of the CP in the PLA fiber mat serves to meet two purposes. First, as it was discussed in the previous section, a small amount of adequately dispersed P3TMA is able to reduce the SE parameters, thus decreasing the wetting behavior and slowing down the rapid drug release in polar solvents. Second, it is expected that cells will respond to an external electrical or electrochemical stimuli when in contact with the $\mathrm{CP}$. Therefore, before drug-loading, the bulk conductivity of PLA-P3TMA fiber mats was evaluated by EIS. Samples were prepared by electrospinning and cut in disc-shaped formats for

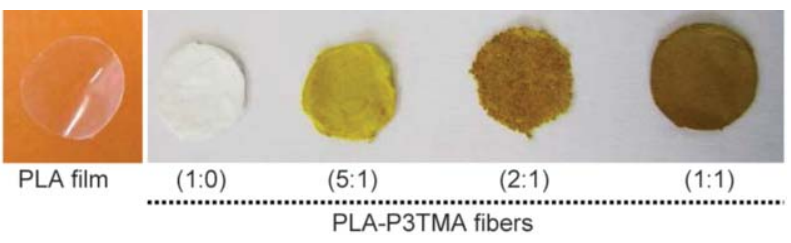

Figure 4. Photographs of PLA-P3TMA and PLA electrospun fibers and PLA saolvent casted films prepared for EIS analyses using a capacitor cell

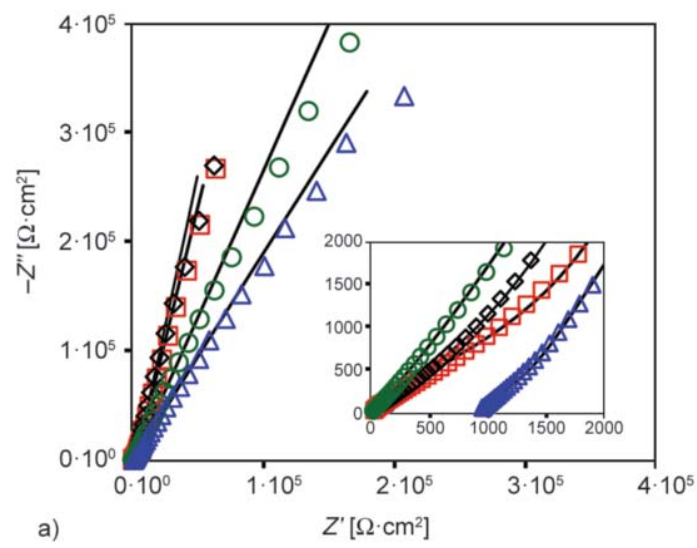

adaptation to the capacitor cell [35]. As it is illustrated in Figure 4, scaffolds become darker with increasing content of $\mathrm{CP}$.

The Nyquist and Bode plots provide information about the distribution of the $\mathrm{CP}$ in the biopolymer matrix. Figure 5a indicates that PLA and 5:1 PLAP3TMA scaffolds exhibit similar resistance and capacitance, indicating that the incorporation of a low concentration of CP does not alter the electrochemical properties of PLA fibers. Results for the three replicated samples of each system were alike and, therefore, only one curve was represented. Spectra show a very compressed and very small beginning of a semicircle in the high frequency range, and an inclined straight line in the low frequency range. It is well known that the high frequency semicircle is associated with the bulk relaxation of the film and its irregular thickness and morphology, while the straight line refers to the migration of ions and the surface inhomogeneity of the electrodes [46].

On the other hand, spectra for the 1:1 PLA-P3TMA scaffolds, which contain the highest P3TMA concentration, show a slightly different behavior as it is observed in the corresponding Bode plot (Figure 5b). The phase angle for samples with such composition is very low in the high frequency region, which only can be explained by the presence of defects caused

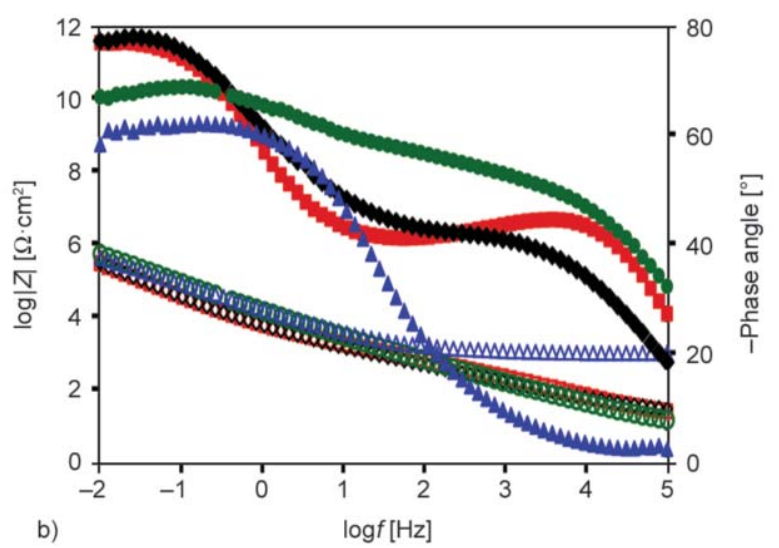

Figure 5. (a) Nyquist plots of PLA (square) and 5:1, 2:1 and 1:1 PLA-P3TMA (diamond, round and triangle, respectively) fiber matrices obtained using a capacitor cell. The inset in the diagram shows the impedance plot with magnified scale axes. Continuous lines correspond to the fitting of results obtained using the EEC to the experimental data. (b) Bode diagram from Nyquist plot. Filled figures refer to the angle phase axis, while empty figures refer to the $\log |Z|$ axis. 
by an irregular distribution of P3TMA aggregates inside the fibers. Such distribution favors the rapid penetration and conduction of the electrolyte across the scaffold. In contrast, P3TMA seems to be homogeneously distributed in 2:1 PLA-P3TMA fibers showing a Nyquist plot with the lowest resistance and capacitance values (Figure 5a), whereas the Bode plot (Figure $5 \mathrm{~b}$ ) presents the highest phase angle $\left(32.1^{\circ}\right)$ and the lowest $\log |Z|(1.15)$ at the high frequencies zone $\left(10^{5} \mathrm{~Hz}\right)$. Quantitative analyses of these results required fitting with an adequate electrical equivalent circuit (EEC) for each system (Figure 6).

In our experiments, a PLA-P3TMA film was placed between two stainless steel (SS) electrodes forming through-plane cell geometry for bulk resistance and capacitance measurements across the film, i.e. forming a capacitor cell [35]. This means that, under polarization, an electrical double layer will be formed at the electrodes interface. This charged ion monolayer includes the electrolyte ions arranged in a monolayer at the electrode surface since they cannot trespass the electrode and the layer of opposite ionic charge at the electrode surface [46]. As it was carefully explained by Soboleva et al. [47], the idealized circuit for such a system has several elements and can be represented by the EEC displayed in Figure 6a. Briefly, each film/blocking electrode interface is represented by the parallel combination of a capacitance, which models the capacitance of the double layer $\left(C_{1}\right.$ and $\left.C_{2}\right)$, and the resistance of the blocking electrode $\left(R_{1}\right.$ and $\left.R_{2}\right)$. Besides, the bulk properties of the polymer film are represented by means of a bulk membrane capacitance $\left(C_{\mathrm{b}}\right)$ and a bulk resist-

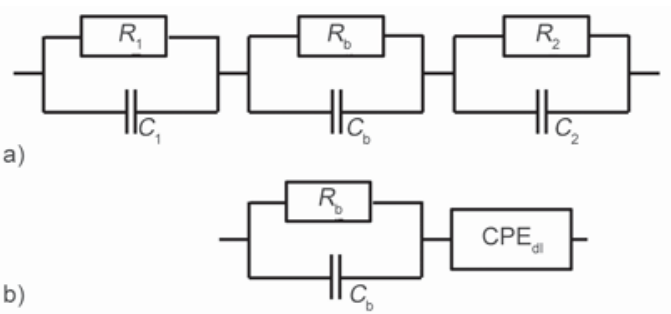

c)

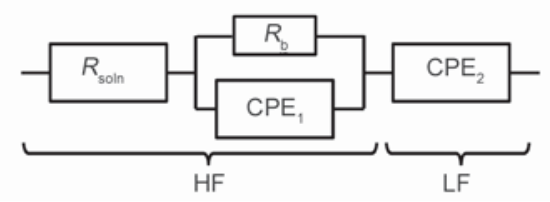

Figure 6. Equivalent electrical circuit for the scaffold films between two blocking electrodes: (a) idealized circuit, (b) simplified circuit and (c) electrical equivalent circuit of PLA-P3TMA system. HF stands for High Frequency range and LF for Low Frequency range. ance $\left(R_{\mathrm{b}}\right)$. However, the above circuit can be simplified into the one shown in Figure $6 \mathrm{~b}$ by taking into account the following considerations: $(i)$ the electrode/electrolyte interface is merely capacitive, therefore resistances $R_{1}$ and $R_{2}$ can be omitted; and (ii) the dominant process at the proton conducting electrode can be considered as a double layer capacitance. The elements of this equivalent electric circuit are the $C_{\mathrm{dl}}$, which models the interfacial capacitance that arises from the double layer capacitance at the film/ electrode interface, and $R_{\mathrm{b}}$ and $C_{\mathrm{b}}$, associated in parallel, which represent the bulk resistance and the geometrical capacitance of the film/electrode interface, respectively.

As the real system is much more complicated and the capacitance at solid electrodes does not behave ideally, two constant phase elements substitute the capacitors $C_{\mathrm{dl}}$ and $C_{\mathrm{b}}$ considering the inhomogeneity of the system and the roughness and irregularities of the electrode surface [48, 49]. After fitting the experimental data to an equivalent electrical circuit it was found that PLA and PLA-P3TMA blends of $w t \%$ ratio 5:1 and 2:1 adjust to the circuit depicted in Figure $6 \mathrm{c}$, while the fitting of a circuit to the $1: 1$ blend was much more difficult to achieve. This has been attributed to the fact that the high concentration of $\mathrm{CP}$ in the 1:1 film provokes the formation of P3TMA agglomerates inside the polymer matrix, resulting in a complex interface distribution. Thus, only the experimental data of one tested sample was properly fitted with the previous circuit. Therefore, in terms of the equivalent circuit elements analysis, the values displayed in Table 4 for the 1:1 blend are not averages. The electrical equivalent circuit used for the fitting of PLA and PLA-P3TMA EIS data was: $R_{\text {soln }}\left(R_{\mathrm{b}} \mathrm{CPE}_{1}\right) \mathrm{CPE}_{2}$. It only differs from the general circuit described above (Figure 6b) in the presence of $R_{\text {soln }}$, which models the PBS electrolyte resistance. The parallel combination of $R_{\mathrm{b}}$ and $\mathrm{CPE}_{1}$ re-

Table 4. Thickness and bulk conductivity values for the PLAP3TMA fiber scaffolds obtained by EIS measurements

\begin{tabular}{|l|c|c|}
\hline \multicolumn{1}{|c|}{$\#$} & $\begin{array}{c}\text { Thickness } \\
{[\boldsymbol{\mu m}]}\end{array}$ & $\begin{array}{c}\boldsymbol{\sigma} \cdot \mathbf{1 0}^{-\mathbf{7}} \\
{\left[\mathbf{S} \cdot \mathbf{c m}^{-\mathbf{1}}\right]}\end{array}$ \\
\hline PLA & $18.9 \pm 4.2$ & $4.33 \pm 0.91^{\mathrm{a}}$ \\
\hline PLA-P3TMA 5:1 & $26.0 \pm 5.9$ & $5.39 \pm 0.72^{\mathrm{a}}$ \\
\hline PLA-P3TMA 2:1 & $25.2 \pm 10.6$ & $8.26 \pm 0.59^{\mathrm{a}}$ \\
\hline PLA-P3TMA 1:1 & $24.8 \pm 5.8$ & $8.16^{\mathrm{b}}$ \\
\hline
\end{tabular}

${ }^{a}$ Average of three independent samples fitted to the proposed electrical equivalent circuit (EEC).

${ }^{b}$ Only one sample fitted properly to the proposed EEC. 
sults in the high frequency semicircle while the $\mathrm{CPE}_{2}$ represents de double layer capacitance. The mean of the simulated values for the EIS data are summarized in Table 4. The CPE impedance can be expressed as is indicated in Equations (1) and (2):

$$
\begin{aligned}
& Z_{\mathrm{CPE}}=B(j \omega)^{-\mathrm{n}} \\
& \alpha=(1-n) \frac{\pi}{2}
\end{aligned}
$$

where $B=C_{\mathrm{d}}^{-1}$ only when $n=1$, and $n$ is related to $\alpha$ which is the deviation from the vertical of the line in the Nyquist plot. Thus, $n=1$ equals an ideal capacitance, and lower $n$ values reflect the roughness of the electrode employed [46].

The EIS results showed that the density of the charge carriers and their mobility increase with the P3TMA content in the scaffold, improving the bulk conductivity. More specifically, the bulk conductivity of the fiber matrix films can be calculated by applying the Equation (3):

$$
\delta=\frac{1}{R_{\mathrm{b}}} \cdot \frac{d}{A}
$$

where $\delta$ is the bulk conductivity, $d$ is the thickness of the fiber film, $A$ is the area of the polymer discs and $R_{\mathrm{b}}$ is the bulk resistance.

The good dispersion of P3TMA particles in the polymer matrix, under electrospinning conditions, is critical to obtain good electrical properties in the isolating fiber blends. Table 5 lists the bulk conductivity for all examined system. Comparison of the results obtained for 2:1 PLA-P3TMA and PLA indicates that the incorporation of $33 \mathrm{wt} \%$ of P3TMA into the fibers composition increases the bulk conductivity by $91 \%$. According to these results, 2:1 PLA-P3TMA fiber mats were chosen for drug-loading and release assays since they accomplish the two-fold characteristic described before: good wettability and the highest bulk conductivity $\left(8.26 \cdot 10^{-7} \mathrm{~S} \cdot \mathrm{cm}^{-1}\right)$.

Bulk conductivities were recently reported for other biodegradable polymer-CP blended systems. For ex- ample, the electrical conductivity of electrospun fibers made of PLA blended with undoped polyaniline (PLA-PAni) and with polyaniline doped with camphorsulfonic acid (PLA-PAni.CSA) was reported to be $1.5 \cdot 10^{-5}$ and $8.4 \cdot 10^{-2} \mathrm{~S} \cdot \mathrm{cm}^{-1}$, respectively [50]. Also, McKeon et al. [51] electrospun several polyaniline and poly(D,L-lactide) (PANi/PDLA) mixtures using different weight percent values. The electrical conductivity of the $75 / 25$ electrospun scaffold, which was the only able to conduct a current of $5 \mathrm{~mA}$, was $4.37 \cdot 10^{-2} \mathrm{~S} \cdot \mathrm{cm}^{-1}$. In another work, PAni $\cdot \mathrm{CSA}$ was blended with poly(L-lactide- $c o$ - $\varepsilon$-caprolactone) (PLCL) to obtain uniform nanofibers with good electrical properties [52]. Recently, incorporation of PAni into polycaprolactone (PCL) fibers significantly increased the electrical conductivity from a non-detectable level for the PCL fibers to $63.6 \cdot 10^{-3} \pm$ $6.6 \cdot 10^{-3} \mathrm{~S} \cdot \mathrm{cm}^{-1}$ for the fibers containing $3 \mathrm{wt} \% \mathrm{PANi}$ [53]. Forciniti et al. [54] suggested that blends of poly(lactic-co-glycolic acid) and chloride-doped PPy exhibit the same electrical properties that the individual CP. Conductive PPy coating polyamide 6 (PA6) nanofibers were prepared by polymerizing pyrrole monomers directly onto the fiber [55].

\subsection{PLA-P3TMA fibers loaded with drugs}

Once the PLA-P3TMA-drug $(2: 1: 0.4 \mathrm{w} / \mathrm{w})$ samples were prepared considering CIP (antibiotic), CHX and TCS (biocides), and IBU (anti-inflammatory), SEM micrographs were taken to identify the possible morphological changes induced by the loading process. The surface of the loaded fibers was found to be less smooth and homogeneous than those of unloaded fiber samples. The lack of uniformity is reflected in Figures $7 \mathrm{a}$ and $7 \mathrm{~b}$ for IBU and CIP drug loaded fibers, respectively, exhibiting enhanced flexibility and non-homogeneous diameters (i.e. varying from 1.58 to $5.09 \mu \mathrm{m}$ ) with respect to unloaded PLAP3TMA. Besides, fibers tend to agglomerate without directional preference forming disorganized branches with diameters between 11 and $40 \mu \mathrm{m}$. Furthermore, the porosity of IBU loaded fibers is very high

Table 5. Data of EIS results obtained from the electrical equivalent circuit showed in Figure 6 for all polymeric blends

\begin{tabular}{|c|c|c|c|c|c|c|}
\hline PLA-P3TMA & $\begin{array}{c}\mathbf{R}_{\text {sol }} \\
{[\boldsymbol{\Omega}]}\end{array}$ & $\begin{array}{c}\mathbf{C P E}_{\mathbf{1}} \\
{\left[\mathbf{F} \cdot \mathbf{s}^{\mathbf{n}-\mathbf{l}}\right]}\end{array}$ & $\boldsymbol{n}_{\mathbf{1}}$ & $\begin{array}{c}\mathbf{R}_{\mathbf{b}} \\
{[\mathbf{k} \boldsymbol{\Omega}]}\end{array}$ & $\begin{array}{c}\mathbf{C P E}_{\mathbf{2}} \\
{\left[\mathbf{F} \cdot \mathbf{s}^{\mathbf{n}-1}\right]}\end{array}$ & $\boldsymbol{n}_{\mathbf{2}}$ \\
\hline $1: 0$ & $8.24 \pm 3.34$ & $5.00 \cdot 10^{-5} \pm 1.71 \cdot 10^{-5}$ & $0.50 \pm 0.08$ & $2.47 \pm 0.09$ & $2.27 \cdot 10^{-5} \pm 1.82 \cdot 10^{-5}$ & $0.73 \pm 0.13$ \\
\hline $5: 1$ & $11.73 \pm 3.91$ & $1.55 \cdot 10^{-4} \pm 3.02 \cdot 10^{-5}$ & $0.51 \pm 0.02$ & $2.74 \pm 0.54$ & $4.21 \cdot 10^{-5} \pm 1.90 \cdot 10^{-6}$ & $0.87 \pm 0.03$ \\
\hline $2: 1$ & $11.62 \pm 5.58$ & $1.47 \cdot 10^{-4} \pm 5.76 \cdot 10^{-5}$ & $0.50 \pm 0.03$ & $1.75 \pm 0.82$ & $1.51 \cdot 10^{-5} \pm 8.83 \cdot 10^{-6}$ & $0.73 \pm 0.05$ \\
\hline $1: 1^{\mathrm{a}}$ & 939 & $1.32 \cdot 10^{-5}$ & 0.66 & 1.456 & $4.88 \cdot 10^{-6}$ & 0.80 \\
\hline
\end{tabular}

${ }^{\mathrm{a}}$ Only one sample properly fitted to the equivalent circuit proposed. 

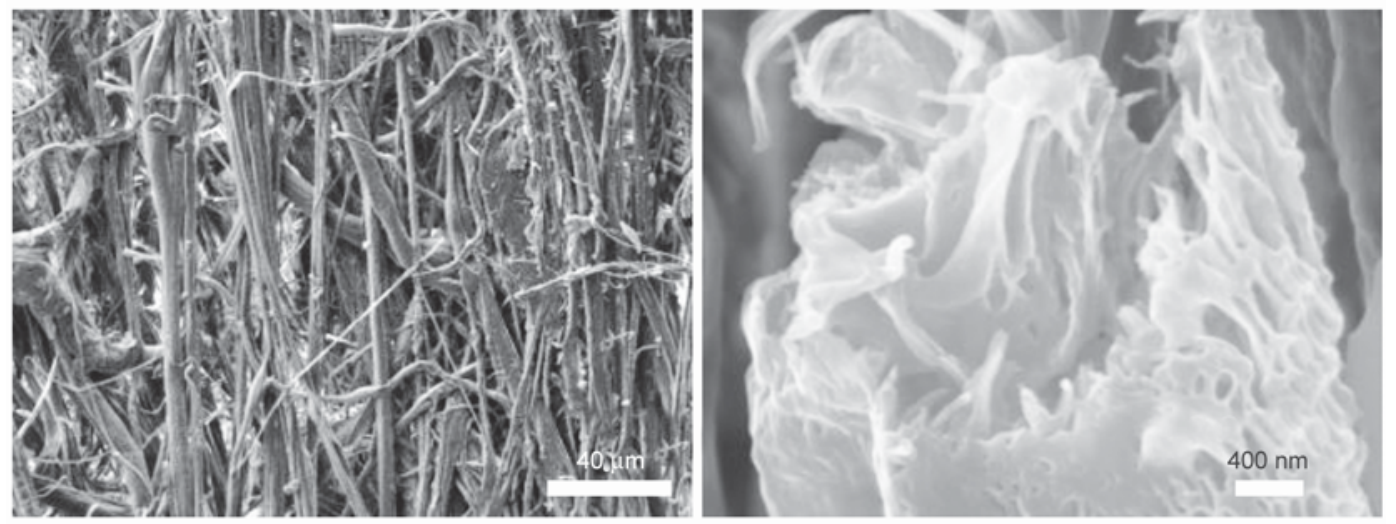

a)
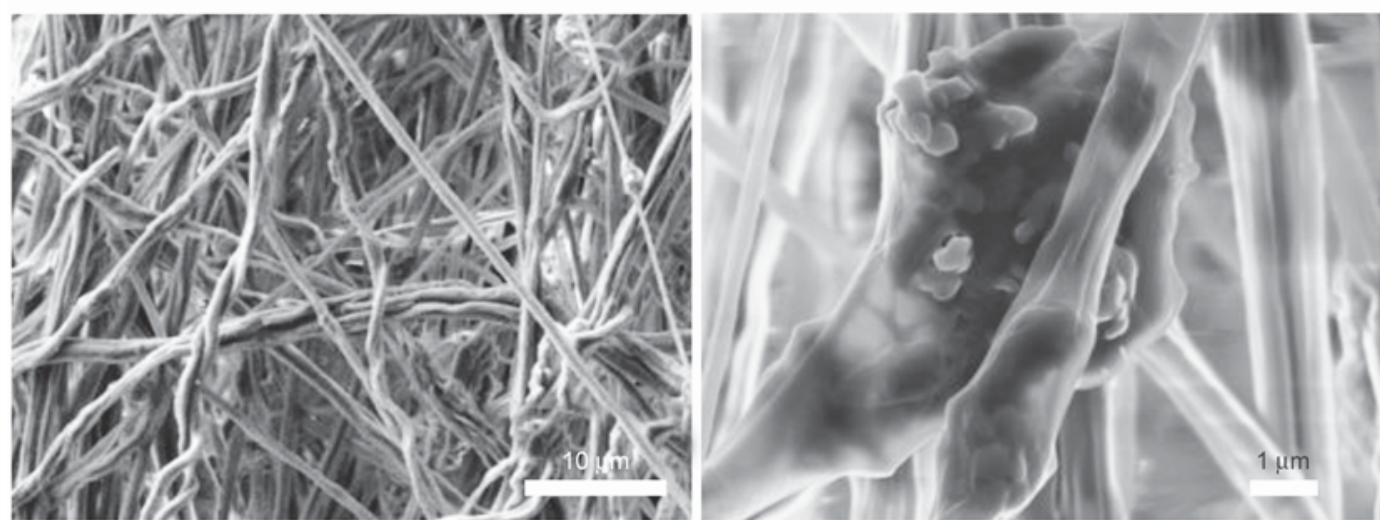

b)
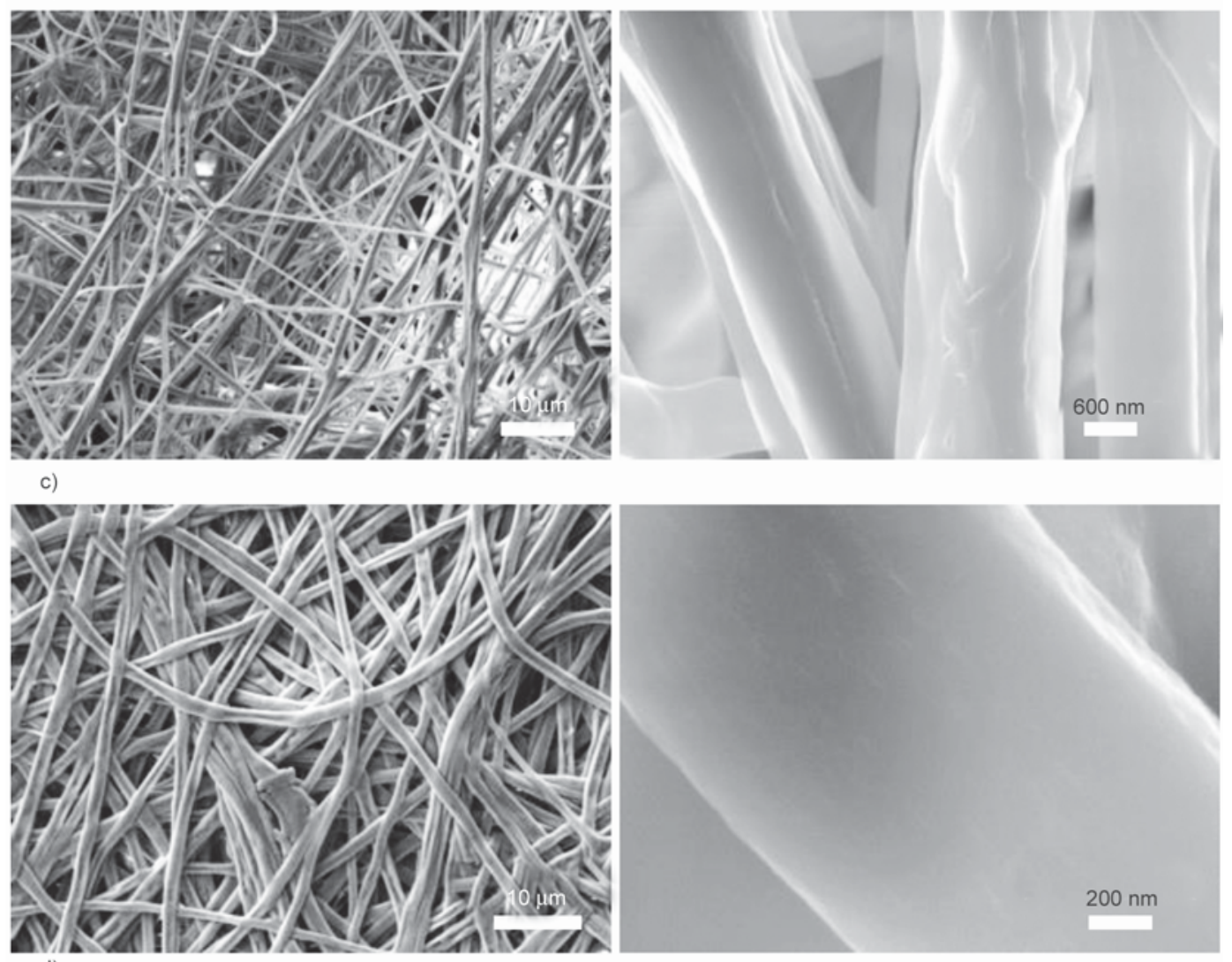

Figure 7. SEM images of 2:1 PLA-P3TMA fibers loaded with: (a) IBU, (b) CIP, (c) CHX and (d) TCS. Left and right images correspond to small and high magnifications. 
in both outside and inside, as is reflected in the micrographs of the interior of broken morphologies (Figure $7 \mathrm{a}$, right). Figure $7 \mathrm{~b}$ (right), which displays a PLA-P3TMA-CIP fiber, allows us to distinguish embedded drug particles.

Morphological changes are less severe for $\mathrm{CHX}$ loaded samples than for IBU- and CIP-containing ones. Thus, well-formed and homogeneous scaffolds made of fibers with morphology, diameter and surface roughness similar to the unloaded ones are obtained (Figure 7c). Finally, TCS-containing samples provide the most homogenous fibers, exhibiting a highly smooth surface that largely resembles the texture of unloaded 2:1 PLA-P3TMA fibers (Figure 7d). In spite of this, AFM images displayed in Figure 8 evidence that TCS provokes a drastic change in the shape of the fibers. Thus, compact and regular PLAP3TMA-TCS fibers present a ribbon-like shape rather than a tubular shape like that observed for $2: 1$ PLA-P3TMA fibers (Figure 1c). Variations in the fiber texture with respect to PLA-P3TMA are reflected in the AFM images of PLA-P3TMA-CHX, PLA-P3TMA-CIP and PLA-P3TMA-IBU, which are displayed in Figure 9. The different effects of

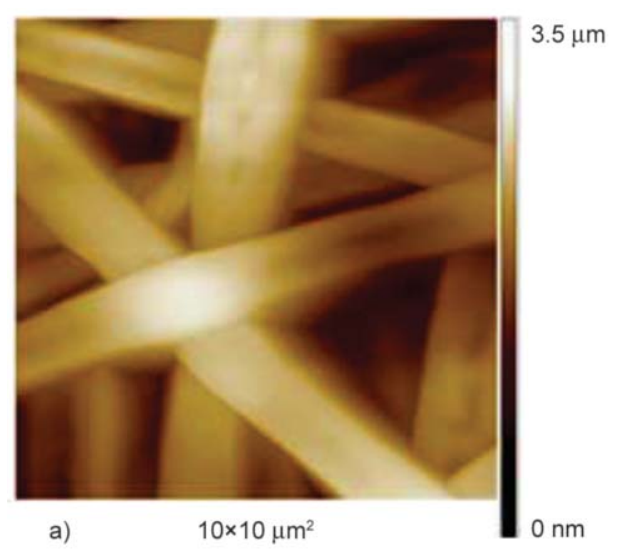

loaded drugs into fiber dimensions have been attributed to the changes induced by drug $\cdots$ drug, drug $\cdots$ PLA and drug ..P3TMA interactions, which alter the polymer $\cdots$ polymer interactions established in unloaded fibers. Thus, attractive and repulsive interactions may provoke the collapse and expansion of the polymeric matrix, respectively. Similarly, the balance between the different interactions is expected to affect the texture and roughness of the fibers.

On the other hand, considering the homogeneity of the electrospinning media, which were obtained by adding the drug dissolved in DMSO to a chloroformacetone mixture containing the two polymers, and the fact SEM and AFM micrographs indicate that the drugs were not located outside the fibers, the drug loading efficiency is considered to be very high in all cases.

Confocal Raman spectroscopy was also used to examine the drug-loading into the PLA-P3TMA samples. Figure 10, which shows the spectrum for the PLA-P3TMA-CIP samples, reflects the presence of several peaks (brown-shadowed) coming from the drug and the main absorption band from P3TMA at about $1480 \mathrm{~cm}^{-1}$. The corresponding optical image

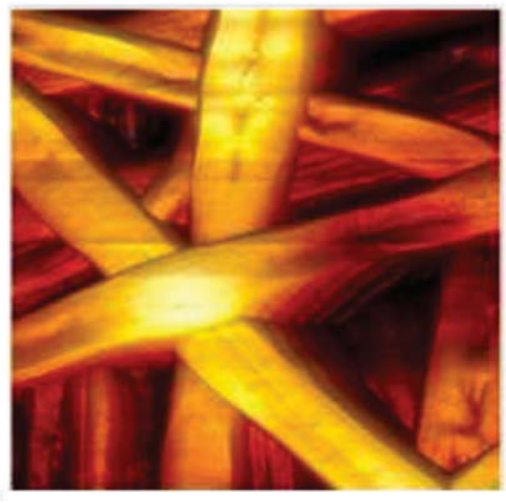

b)

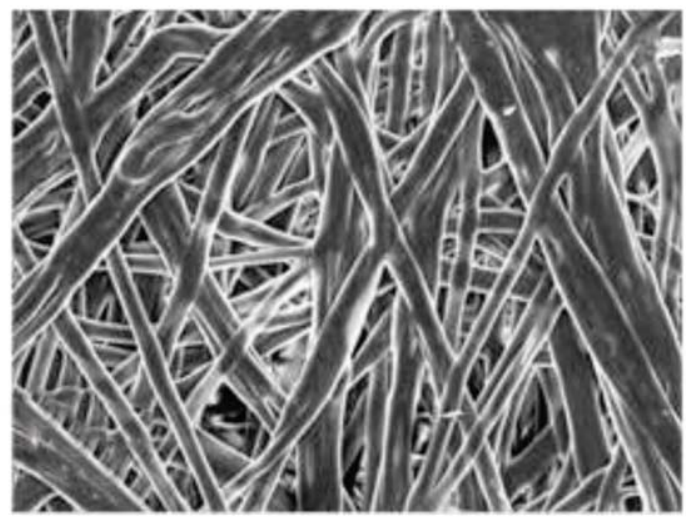

c)

$4 \mu \mathrm{m}$

Figure 8. AFM micrographs from PLA-P3TMA-TCS fibers: (a) 2D height image, (b) 3D image $\left(10 \times 10 \mu \mathrm{m}^{2}\right)$ and (c) SEM micrograph (scale bar: $4 \mu \mathrm{m}$ ) 

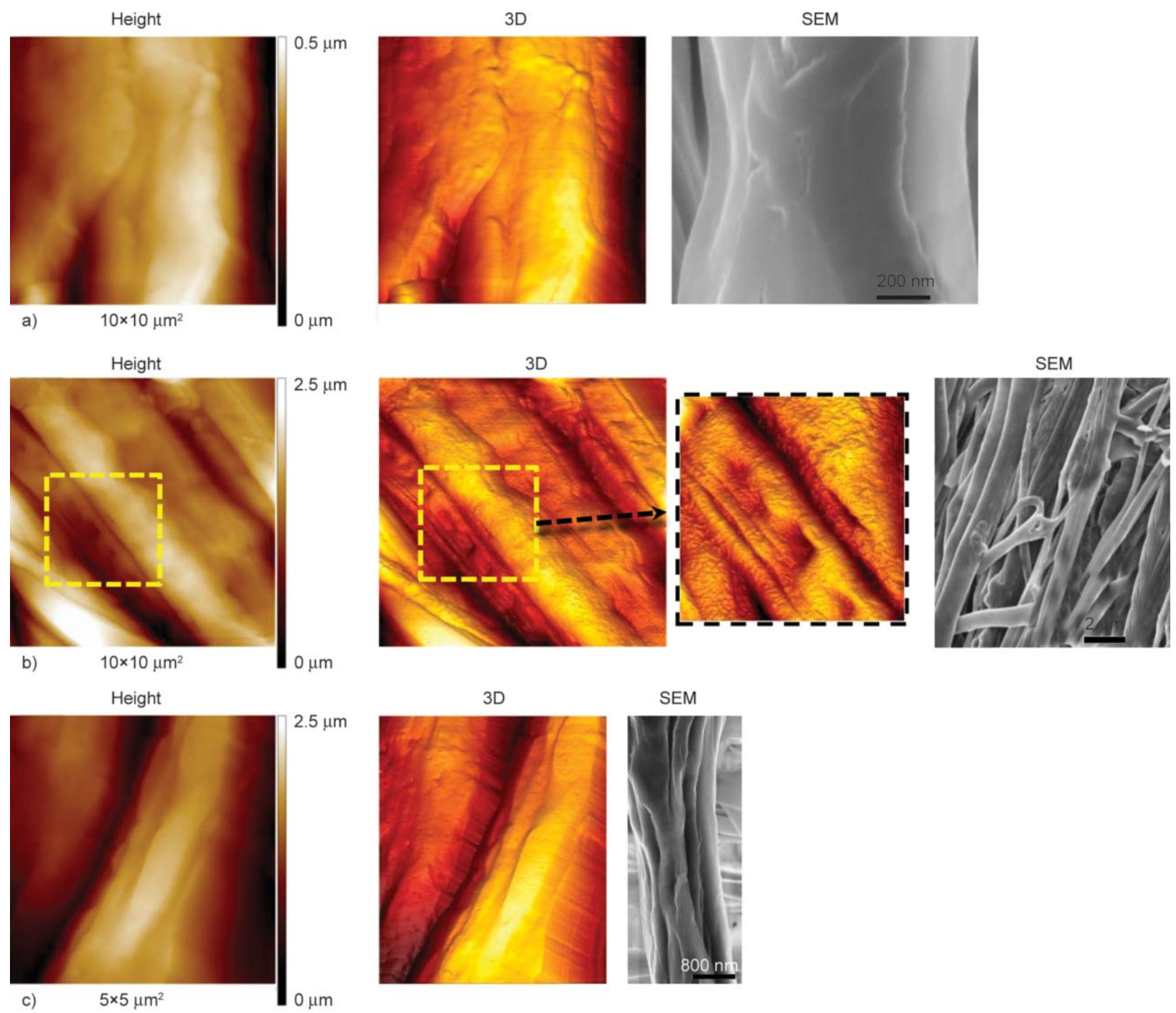

Figure 9. AFM micrographs from PLA:P3TMA 2:1 loaded with CHX (a), IBU (b) and CIP (c): 2D height image, 3D images and SEM micrograph

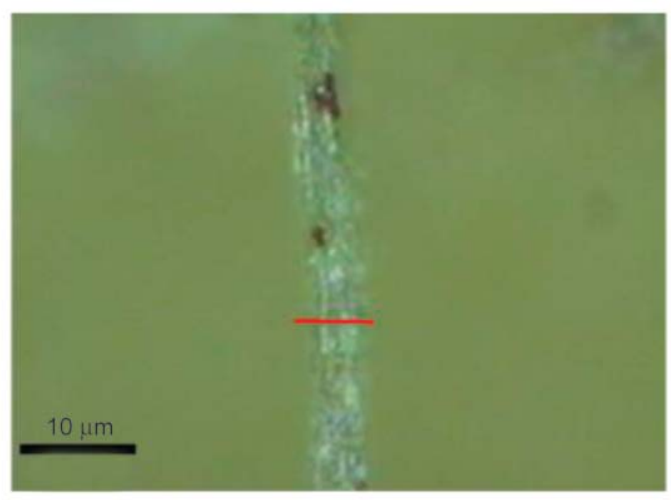

a)

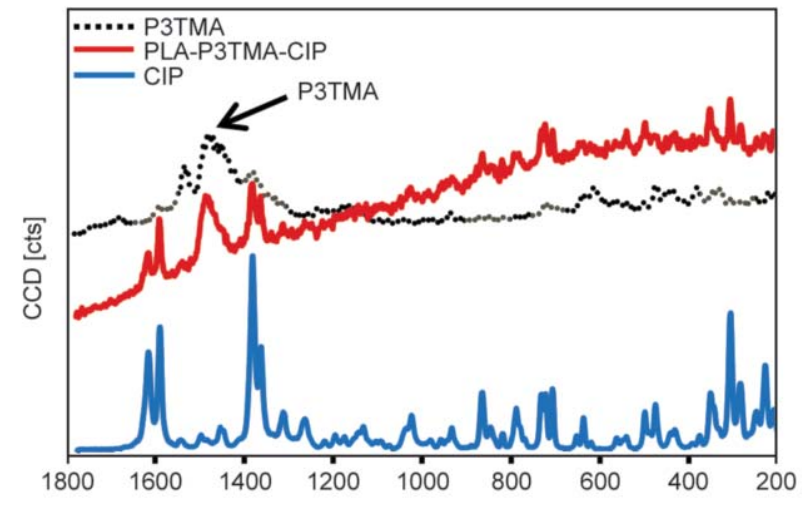

b)

$$
\text { Rel. }[1 / \mathrm{cm}]
$$

Figure 10. (a) Optical image of PLA-P3TMA-CIP fibers. The red line shows the spot in which the Raman spectrum was taken. (b) Raman spectra: the dotted line corresponds to P3TMA powder, the blue line to CIP powder and the red line to the PLA-P3TMA-CIP sample.

displays a white shine along the fiber that has been attributed to the drug. Unfortunately, identification of the loaded drug was less clear for the other three cases (not shown; available upon request to the authors), which has been attributed to its small concentration (12 wt \%). 


\subsection{Drug release study}

Drug release from electrospun fibers in a given medium usually relates to: $(i)$ the morphology and crystallinity of such fibers; and (ii) the intermolecular interactions established between the drugs and the polymeric matrix. Within this context, it should be noted that the crystallinity and thermal behavior of PLA-P3TMA fibers was previously examined [33]. Quantitative release studies were performed considering PLA-P3TMA matrices loaded with CIP, CHX, TCS and IBU. Both PBS and PBS-EtOH were used as release media. According to previous studies [36], the ethanol supplement facilitates the delivery of highly hydrophobic molecules, such as TCS and IBU, precluding the establishment of early equilibrium conditions that limit their release in PBS. Figure 11a shows the release profiles in PBS for all four drugs loaded in PLA-P3TMA. The lower values were obtained for TCS (30\%) and IBU (25\%), suggesting that the retention into the polymer crystalline domains was higher for these drugs than for CIP and CHX. However, the release of TCS and IBU increases to 90 and $80 \%$, respectively, when the delivery was performed using the more hydrophobic PBSEtOH environment (Figure 11b).

On the other hand, release percentages in PBS of CIP and CHX from loaded PLA-P3TMA (i.e. $~ 90$ and $\sim 70 \%$, respectively) are higher than those obtained for TCS and IBU. Furthermore, the release of CHX in PBS-EtOH was also very fast, reaching a value of $\sim 70 \%$ after only seven hours of exposure (Figure 11b). Previous studies [56-58] indicated that the release kinetics of these drugs can be explained by the Higuchi and first-order models, which are usu-

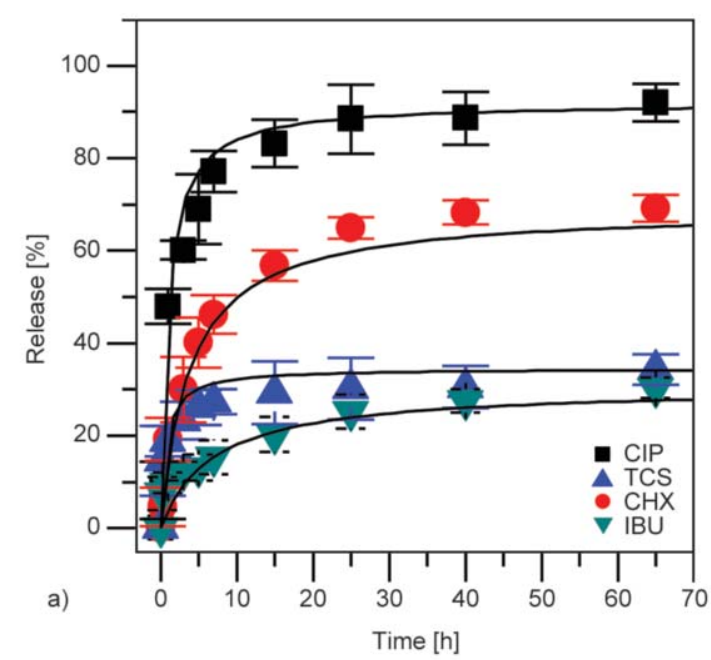

ally combined to describe the first (from the beginning to $60 \%$ ) and second (40-100\%) stages of the release [59], respectively. In both cases, the delivery process is explained by the diffusion of the drug through the polymer matrix, which is facilitated by the ethanol-induced swelling of PLA [36].

Accordingly, the release profiles of CIP, CHX, TCS and IBU drugs loaded in the PLA-P3TMA scaffolds allowed us to reach some important conclusions: a) the electrospun hybrid fibers of PLA and P3TMA can be successfully used to load either hydrophilic and hydrophobic drugs; and $b$ ) the behavior of loaded 2:1 PLA-P3TMA fibers depends on the release environment, drug release being faster in a more hydrophobic medium.

\subsection{Evaluation of bactericide activity of $2: 1$ PLA-P3TMA-drug scaffolds}

Agar diffusion tests were conducted to demonstrate qualitatively the biological activity of the antimicrobial drugs (CIP, CHX, and TCS) loaded into 2:1 PLAP3TMA electrospun fibers (Figure 12). E. coli and $S$. epidermidis bacteria were chosen as representative Gram negative and Gram positive bacteria groups, respectively. There was no inhibition halo in the growth plate of E. coli and S. epidermidis (Figures 12a and $12 \mathrm{~b}$, respectively) around the unloaded and IBUloaded PLA-P3TMA samples. This expected result is consistent with the lack of bactericidal activity of PLA, P3TMA and IBU.

In contrast, halos of inhibition were observed for PLA-P3TMA electrospun fibers loaded with TCS, $\mathrm{CHX}$ and CIP, evidencing the antimicrobial activity of these drugs $[36,56,57,60]$. The formation of such

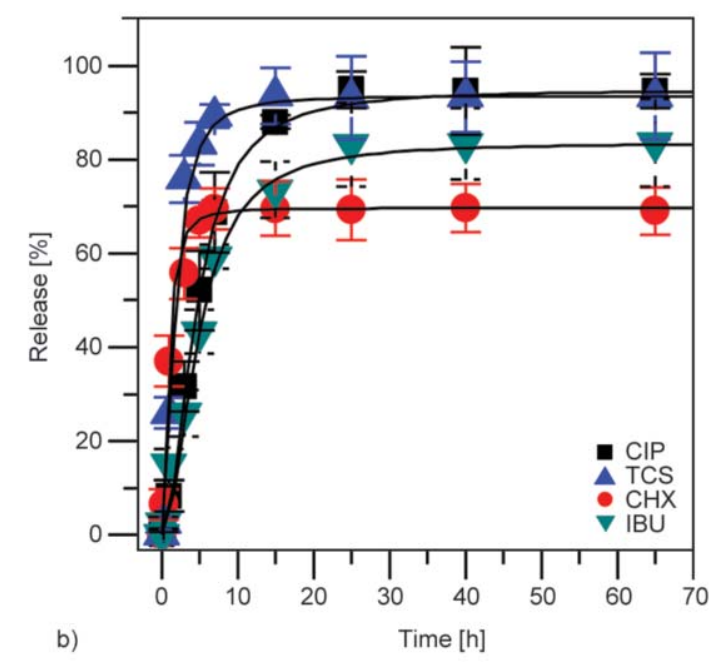

Figure 11. Drug release profile from 2:1 PLA-P3TMA scaffolds in (a) PBS and (b) PBS-EtOH 

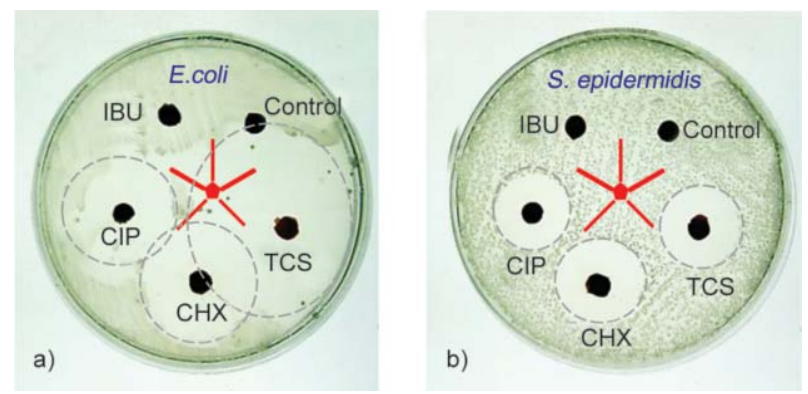

Figure 12. Inhibition of bacteria growth for (a) Escherichia coli and (b) Staphylococcus epidermidis. Unloaded 2:1 PLA-P3TMA scaffold was used as control disk. TCS, CHX, CIP and IBU disks refer to 2:1 PLA-P3TMA fibers loaded with the corresponding drug. Red lines indicate the areas of the plates occupied by each samples. The inhibition zones are highlighted with dotted lines.

halos corresponds to the drug released from the scaffold and, subsequently, diffused through the agar. The diameters of the three halos displayed in Figure $12 \mathrm{~b}$ are similar, suggesting that the susceptibility of $S$. epidermidis towards TCS, CHX and CIP is similar. However, the response of E. coli towards such drugs is completely different, as it is reflected by the different sizes of the halos in Figure 12a. Although the agar diffusion test can also be used as a quantitative method, the diameter of the halos is only indicative of bacterial susceptibility towards the drug. In summary, these results clearly prove that the biological activity of the drugs is not affected by the electrospinning process or by the formation of interactions between the drugs and the PLA-P3TMA matrix.

\section{Conclusions}

Fibers made of PLA and P3TMA, a biopolymer and a $\mathrm{CP}$, respectively, have been prepared by electrospinning and, subsequently, characterized. The first step consisted in the optimization of the electrospinning parameters, surface and conducting properties of fibers prepared using 5:1, 2:1 and 1:1 PLA-P3TMA compositions being examined. After this, fibers were loaded with different drugs and both their release and activity were studied.

Our results demonstrate that the incorporation of P3TMA into PLA scaffolds provokes changes in the surface morphology and decreases the bulk resistance. Fibers prepared using 2:1 PLA-P3TMA exhibited an increment in the bulk conductivity of $\sim 90 \%$ in comparison to PLA. The observed semiconducting behavior suggests that scaffolds prepared using such composition would be appropriate for the development of electrically stimulated drug-delivery systems. Furthermore, wettability measurements prove that P3TMA reduces the surface energy parameters of PLA. This has been shown to improve the applicability of the prepared fibers as drug delivery systems.

Drug release experiments in PBS and PBS-EtOH with 2:1 PLA-P3TMA-drug scaffolds proved that the release kinetics depends on the hydrophilicity of solution medium, being faster for the least hydrophilic medium. Moreover, CHX showed a stable release behavior either in PBS or in PBS-EtOH solutions $(65-70 \%)$, combined with a good fiber formation by electrospinning. As it was expected, unloaded fibers (blank samples) do not show antibacterial activity against $E$. coli and $S$. epidermidis bacteria. However, PLA-P3TMA electrospun matrices loaded with TCS, CHX and CIP drugs showed normal activity against such two bacteria, proving that the bioactivity of these drugs was not altered by the electrospinning process. Overall, these results demonstrate that the drugs are well mixed in the PLA-P3TMA scaffold matrices. Future work is oriented towards the release of drugs loaded on PLA-CP fibers by electro stimulation.

\section{Acknowledgements}

Authors are indebted to supports from MICINN and FEDER (MAT2012-34498 and MAT2012-36205). M.M.P.-M. thanks financial support through a FPI-UPC grant. Support for the research of C.A. was received through the prize 'ICREAAcademia' for excellence in research funded by the Generalitat de Catalunya.

\section{References}

[1] Okada H., Toguchi H.: Biodegradable microspheres in drug delivery. Critical Reviews in Therapeutic Drug Carrier Systems, 12, 1-99 (1995).

DOI: $10.1615 /$ CritRevTherDrugCarrierSyst.v12.i1.10

[2] Kitchell J., Wise D.: Poly(lactic/glycolic acid) biodegradable drug-polymer matrix systems. Methods in Enzymology, 112, 436-448 (1985).

DOI: $10.1016 / \mathrm{S} 0076-6879(85) 12034-3$

[3] Kane J. M., Eerdekens M., Lindenmayer J-P., Keith S. J., Lesem M., Karcher K.: Long-acting injectable risperidone: Efficacy and safety of the first long-acting atypical antipsychotic. The American Journal of Psychiatry, 160, 1125-1132 (2003). DOI: 10.1176/appi.ajp.160.6.1125 
[4] Hans M., Shimoni K., Danino D., Siegel S. J., Lowman A.: Synthesis and characterization of mPEG-PLA prodrug micelles. Biomacromolecules, 6, 2708-2717 (2005). DOI: $10.1021 / \mathrm{bm} 050188 \mathrm{k}$

[5] Pérez-Madrigal M. M., Armelin E., Puiggalí J., Alemán C.: Insulating and semiconducting polymeric free-standing nanomembranes with biomedical applications. Journal of Materials Chemistry B, 3, 5904-5932 (2015). DOI: $10.1039 / \mathrm{c} 5 \mathrm{tb} 00624 \mathrm{~d}$

[6] Widmer M. S., Gupta P. K., Lu L., Meszlenyi R. K., Evans G. R. D., Brandt K., Savel T., Gukrlek A., Patrick C. W., Mikos A. G.: Manufacture of porous biodegradable polymer conduits by an extrusion process for guided tissue regeneration. Biomaterials, 19, 1945-1955 (1998). DOI: 10.1016/S0142-9612(98)00099-4

[7] Rangappa N., Romero A., Nelson K. D., Eberhart R. C., Smith G. M.: Laminin-coated poly(L-lactide) filaments induce robust neurite growth while providing directional orientation. Journal of Biomedical Materials Research, 51, 625-634 (2000).

DOI: 10.1002/1097-4636(20000915)51:4<625::AIDJBM10>3.0.CO;2-U

[8] Wei Y., Lelkes P. I., MacDiarmid A. G., Guterman E., Cheng S., Palouian K.: Electroactive polymers and nanostructured materials for neural tissue engineering. in 'Contemporary topics in advanced polymer science and technology’ (eds.: Zhou Q. F., Cheng S. Z. D.) Peking University Press, Beijing, 430-436 (2004).

[9] Bidez P., Li S., MacDiarmid A. G., Venancio E. C., Wei Y., Lelkes P. I.: Polyaniline, an electroactive polymer, supports adhesion and proliferation of cardiac myoblasts. Journal of Biomaterials Science, Polymer Edition, 17, 199-212 (2006).

DOI: $10.1163 / 156856206774879180$

[10] Kotwal A., Schmidt C. E.: Electrical stimulation alters protein adsorption and nerve cell interactions with electrically conducting biomaterials. Biomaterials, 22, 1055 1064 (2001).

DOI: 10.1016/S0142-9612(00)00344-6

[11] Valentini R. F.: Nerve guidance channels. in 'The biomedical engineering handbook' (ed.: Bronzino J. D.) CRC Press, Boca Raton, Section XIII, Chapter 135, p.13 (2000).

[12] Lee J. Y., Bashur C. A., Milroy C. A., Forciniti L., Goldstein A. S., Schmidt C. E.: Nerve growth factor-immobilized electrically conducting fibrous scaffolds for potential use in neural engineering applications. IEEE Transactions on NanoBioscience, 11, 15-21 (2012).

DOI: 10.1109/TNB.2011.2159621

[13] Gizdavic-Nikolaidis M., Ray S., Bennett J. R., Easteal A. J., Cooney R. P.: Electrospun functionalized polyaniline copolymer-based nanofibers with potential application in tissue engineering. Macromolecular Bioscience, 10, 1424-1431 (2010). DOI: 10.1002/mabi.201000237
[14] del Valle L. J., Estrany F., Armelin E., Oliver R., Alemán C.: Cellular adhesion, proliferation and viability on conducting polymer substrates. Macromolecular Bioscience, 8, 1144-1151 (2008). DOI: $10.1002 / \mathrm{mabi} .200800101$

[15] Schmidt C. E., Shastri V. R., Vacanti J. P., Langer R.: Stimulation of neurite outgrowth using an electrically conducting polymer. Proceedings of the National Academy of Sciences of the United States of America, 94, 8948-8953 (1997).

DOI: $10.1073 /$ pnas.94.17.8948

[16] Svirskis D., Travas-Sejdic J., Rodgers A., Garg S.: Electrochemically controlled drug delivery based on intrinsically conducting polymers. Journal of Controlled Release, 146, 6-15 (2010).

DOI: $10.1016 / \mathrm{j} . j$ conrel.2010.03.023

[17] Bidan G., Lopez C., Mendes-Viegas F., Vieil E.: Incorporation of sulphonated cyclodextrins into polypyrrole: An approach for the electro-controlled delivering of neutral drugs. Biosensors and Bioelectronics, 10, 219229 (1994). DOI: 10.1016/0956-5663(95)96808-C

[18] Richardson R. T., Wise A. K., Thompson B. C., Flynn B. O., Atkinson P. J., Fretwell N. J., Fallon J. B., Wallace G. G., Shepherd R. K., Clark G. M., O'leary S. J.: Polypyrrole-coated electrodes for the delivery of charge and neurotrophins to cochlear neurons. Biomaterials, 30, 2614-2624 (2009).

DOI: $10.1016 /$ j.biomaterials.2009.01.015

[19] Guimard N. K., Gomez N., Schmidt C. E.: Conducting polymers in biomedical engineering. Progress in Polymer Science, 32, 876-921 (2007).

DOI: $10.1016 /$ j.progpolymsci.2007.05.012

[20] Guo B., Glavas L., Albertsson A-C.: Biodegradable and electrically conducting polymers for biomedical applications. Progress in Polymer Science, 38, 1263-1286 (2013).

DOI: 10.1016/j.progpolymsci.2013.06.003

[21] Guo B., Sun Y., Finne-Wistrand A., Mustafa K., Albertsson A-C.: Electroactive porous tubular scaffolds with degradability and non-cytotoxicity for neural tissue regeneration. Acta Biomaterialia, 8, 144-153 (2012).

DOI: $\underline{10.1016 / \text { j.actbio.2011.09.027 }}$

[22] Armelin E., Gomes A. L., Pérez-Madrigal M. M., Puiggalí J., Franco L., del Valle L. J., Rodríguez-Galán A., Campos J. S. C., Ferrer-Anglada N., Alemán C.: Biodegradable free-standing nanomembranes of conducting polymer: Polyester blends as bioactive platforms for tissue engineering. Journal of Materials Chemistry, 22, 585-594 (2012).

DOI: $10.1039 / \mathrm{C} 1 \mathrm{JM} 14168 \mathrm{~F}$

[23] Pérez-Madrigal M. M., Armelin E., del Valle L. J., Estrany F., Alemán C.: Bioactive and electroactive response of flexible polythiophene: Polyester nanomembranes for tissue engineering. Polymer Chemistry, 3, 979-991 (2012). DOI: $10.1039 / \mathrm{c} 2 \mathrm{py} 00584 \mathrm{k}$ 
[24] Pérez-Madrigal M. M., Giannotti M. I., del Valle L. J., Franco L., Armelin E., Puiggalí, J., Sanz F., Alemán C.: Thermoplastic polyurethane: Polythiophene nanomembranes for biomedical and biotechnological applications. ACS Applied and Material Interfaces, 6, 97199732 (2014).

DOI: $10.1021 / \mathrm{am} 502150 \mathrm{q}$

[25] Planellas M., Pérez-Madrigal M. M., del Valle L. J., Kobauri S., Katsarava R., Alemán C., Puiggalí J.: Microfibres of conducting polythiophene and biodegradable poly(ester urea) for scaffolds. Polymer Chemistry, 6, 925-937 (2015).

DOI: $10.1039 / \mathrm{C} 4 \mathrm{PY} 01243 \mathrm{G}$

[26] Guimard N. K. E., Sessler J. L., Schmidt C. E.: Toward a biocompatible and biodegradable copolymer incorporating electroactive oligothiophene units. Macromolecules, 42, 502-511 (2009).

DOI: $10.1021 / \mathrm{ma} 8019859$

[27] Zhao W., Glavas L., Odelius K., Edlund U., Albertsson A-C.: Facile and green approach towards electrically conductive hemicellulose hydrogels with tunable conductivity and swelling behavior. Chemistry of Materials, 26, 4265-4273 (2014).

DOI: $10.1021 / \mathrm{cm} 501852 \mathrm{w}$

[28] Glavas L., Odelius K., Albertsson A-C.: Induced redox responsiveness and electroactivity for altering the properties of micelles without external stimuli. Soft Matter, 10, 4028-4036 (2014).

DOI: $10.1039 / C 4 S M 00258 \mathrm{~J}$

[29] Shi G., Rouabhia M., Wang Z., Dao L., Zhang Z.: A novel electrically conductive and biodegradable composite made of polypyrrole nanoparticles and polylactide. Biomaterials, 25, 2477-2488 (2004).

DOI: 10.1016/j.biomaterials.2003.09.032

[30] Xu H., Holzwarth J. M., Yan Y., Xu P., Zheng H., Yin Y., Li S., Ma P. X.: Conductive PPY/PDLLA conduit for peripheral nerve regeneration. Biomaterials, 35, 225 235 (2014).

DOI: $10.1016 /$ j.biomaterials.2013.10.002

[31] Jeong S. I., Jun I. D., Choi M. J., Nho Y. C., Lee Y. M., Shin H.: Development of electroactive and elastic nanofibers that contain polyaniline and poly(L-lactide- $c o-\varepsilon$ caprolactone) for the control of cell adhesion. Macromolecular Bioscience, 8, 627-637 (2008).

DOI: 10.1002/mabi.200800005

[32] Abidian M. R., Kim D-H., Martin D. C.: Conductingpolymer nanotubes for controlled drug release. Advanced Materials, 18, 405-409 (2006).

DOI: $10.1002 / \mathrm{adma} .200501726$

[33] Llorens E., Pérez-Madrigal M. M., Armelin E., del Valle L. J., Puiggalí J., Alemán C.: Hybrid nanofibers from biodegradable polylactide and polythiophene for scaffolds. RSC Advances, 4, 15245-15255 (2014).

DOI: $10.1039 / \mathrm{C} 3 \mathrm{RA} 42829 \mathrm{~J}$
[34] Kim B., Chen L., Gong J., Osada Y.: Titration behavior and spectral transitions of water-soluble polythiophene carboxylic acids. Macromolecules, 32, 3964-3969 (1999). DOI: $10.1021 / \mathrm{ma} 981848 \mathrm{z}$

[35] Müller F., Ferreira C. A., Azambuja D. S., Alemán C., Armelin E.: Measuring the proton conductivity of ionexchange membranes using electrochemical impedance spectroscopy and through-plane cell. Journal of Physical Chemistry B, 118, 1102-1112 (2014).

DOI: $10.1021 / j p 409675 z$

[36] del Valle L. J., Camps R., Díaz A., Franco L., Rodríguez-Galán A., Puiggalí J.: Electrospinning of polylactide and polycaprolactone mixtures for preparation of materials with tunable drug release properties. Journal of Polymer Research, 18, 1903-1917 (2001). DOI: $10.1007 / \mathrm{s} 10965-011-9597-3$

[37] Kister G., Cassanas G., Vert M.: Effects of morphology, conformation and configuration on the IR and Raman spectra of various poly(lactic acid)s. Polymer, 39, 267273 (1998).

DOI: $10.1016 / \mathrm{S} 0032-3861(97) 00229-2$

[38] Shi G., Xu J., Fu M.: Raman spectroscopic and electrochemical studies on the doping level changes of polythiophene films during their electrochemical growth processes. Journal of Physical Chemistry B, 106, 288292 (2002). DOI: 10.1021/jp013023o

[39] Marmur A.: Wetting on hydrophobic rough surfaces: To be heterogeneous or not to be? Langmuir, 19, 83438348 (2003). DOI: $10.1021 / 1 \mathrm{a} 0344682$

[40] Kaplan J. A., Lei H., Liu R., Padera R., Colson Y. L., Grinstaff M. W.: Imparting superhydrophobicity to biodegradable poly(lactide-co-glycolide) electrospun meshes. Biomacromolecules, 15, 2548-2554 (2014).

DOI: $\underline{10.1021 / \mathrm{bm} 500410 \mathrm{~h}}$

[41] Neumann A. W., Good R. J., Hope C. J., Sejpal M.: An equation-of-state approach to determine surface tensions of low-energy solids from contact angles. Journal of Colloid and Interface Science, 49, 291-304 (1974). DOI: 10.1016/0021-9797(74)90365-8

[42] Fowkes F. M.: Additivity of intermolecular forces at interfaces. I. Determination of the contribution to surface and interfacial tensions of dispersion forces in various liquids. Journal of Physical Chemistry, 67, 2538-2541 (1963).

DOI: $10.1021 / \mathrm{j} 100806 \mathrm{a} 008$

[43] Owens D. K., Wendt R. C.: Estimation of the surface free energy of polymers. Journal of Applied Polymer Science, 13, 1741-1747 (1969). DOI: 10.1002/app.1969.070130815

[44] Kaelble D. H.: Dispersion-polar surface tension properties of organic solids. The Journal of Adhesion, 2, 6681 (1970). DOI: $10.1080 / 0021846708544582$ 
[45] Singh A., Naskar A. K., Haynes D., Drews M. J., Smith Jr. D. W.: Synthesis, characterization and surface properties of poly(lactic acid)-perfluoropolyether block copolymers. Polymer International, 60, 507-516 (2010). DOI: $10.1002 /$ pi.2982

[46] Quian X., Gu N., Cheng Z., Yang X., Wang E., Dong S.: Impedance study of $(\mathrm{PEO})_{10} \mathrm{LiClO}_{4}-\mathrm{Al}_{2} \mathrm{O}_{3}$ composite polymer electrolyte with blocking electrodes. Electrochimica Acta, 46, 1829-1836 (2001). DOI: 10.1016/S0013-4686(00)00723-4

[47] Soboleva T., Xie Z., Shi Z., Tsang E., Navessin T., Holdcroft S.: Investigation of the through-plane impedance technique for evaluation of anisotropy of proton conducting polymer membranes. Journal of Electroanalytical Chemistry, 622, 145-152 (2008).

DOI: 10.1016/j.jelechem.2008.05.017

[48] Rodrigues I. R., de Camargo Forte M. M., Azambuja D. S., Castagno K. R. L.: Synthesis and characterization of hybrid polymeric networks (HPN) based on polyvinyl alcohol/chitosan. Reactive and Functional Polymers, 67, 708-715 (2007).

DOI: $10.1016 /$ j.reactfunctpolym.2007.05.010

[49] Douglass Jr. E. F., Driscoll P. F., Liu D., Burnham N. A., Lambert C. R., McGimpsey W. G.: Effect of electrode roughness on the capacitive behavior of self-assembled monolayers. Analytical Chemistry, 80, 7670 7677 (2008)

DOI: $10.1021 / \mathrm{ac} 800521 \mathrm{z}$

[50] Peng S., Zhu P., Wu Y., Mhaisalkar S. G., Ramakrishna S.: Electrospun conductive polyaniline-polylactic acid composite nanofibers as counter electrodes for rigid and flexible dye-sensitized solar cells. RSC Advances, 2, 652-657 (20012). DOI: $10.1039 / \mathrm{C} 1 \mathrm{RA} 00618 \mathrm{E}$

[51] McKeon K. D., Lewis A., Freeman J. W.: Electrospun poly(D,L-lactide) and polyaniline scaffold characterization. Journal of Applied Polymer Science, 115, 15661572 (2010).

DOI: $10.1002 / a p p .31296$

[52] Jeong S. I., Jun I. D., Choi M. J., Nho Y. C., Lee Y. M., Shin H.: Development of electroactive and elastic nanofibers that contain polyaniline and poly(L-lactide-co$\varepsilon$-caprolactone) for the control of cell adhesion. Macromolecular Bioscience, 8, 627-637 (2008).

DOI: 10.1002/mabi.200800005
[53] Chen M-C., Sun Y-C., Chen Y-H.: Electrically conductive nanofibers with highly oriented structures and their potential application in skeletal muscle tissue engineering. Acta Biomaterialia, 9, 5562-5572 (2013).

DOI: $10.1016 /$ j.actbio.2012.10.024

[54] Forciniti L., Guimard N. K., Lee S., Schmidt C. E.: Unique electrochemically synthesized polypyrrole: Poly (lactic-co-glycolic acid) blends for biomedical applications. Journal of Materials Chemistry, 20, 8865-8874 (2010).

DOI: 10.1039/C0JM01015D

[55] Granato F., Bianco A., Bertarelli C., Zerbi G.: Composite polyamide 6/polypyrrole conductive nanofibers. Macromolecular Rapid Communications, 30, 453-458 (2009). DOI: $10.1002 /$ marc. 200800623

[56] Zurita R., Puiggalí J., Rodríguez-Galán A.: Triclosan release from coated polyglycolide threads. Macromolecular Bioscience, 6, 58-69 (2006).

DOI: $10.1002 /$ mabi.200500147

[57] del Valle L. J., Roa M., Díaz A., Casas M. T., Puiggalí J., Rodríguez-Galán A.: Electrospun nanofibers of a degradable poly(ester amide). Scaffolds loaded with antimicrobial agents. Journal of Polymer Research, 19, 9792-9805 (2012).

DOI: $10.1007 / \mathrm{s} 10965-011-9792-2$

[58] del Valle L. J., Roca D., Franco L., Puiggalí J., Rodríguez-Galán A.: Preparation and release study of ibuprofen-loaded porous matrices of a biodegradable poly(ester amide) derived from L-alanine units. Journal of Applied Polymer Science, 122, 1953-1967 (2012).

DOI: 10.1002/app.34017

[59] Baker R.: Controlled release of biologically active agents. Wiley, New York (1987).

[60] Bottino M. C., Kamocki K., Yassen G. H., Platt J. A., Vail M. M., Ehrlich Y., Spolnik K. J., Gregory R. L.: Bioactive nanofibrous scaffolds for regenerative endodontics. Journal of Dental Research, 92, 963-969 (2013). DOI: $10.1177 / 0022034513505770$ 